\title{
Orbital magnetism of graphene nanostructures: Bulk and confinement effects
}

\author{
Lisa Heße and Klaus Richter \\ Institut für Theoretische Physik, Universität Regensburg, D-93040 Regensburg, Germany \\ (Received 29 April 2014; revised manuscript received 22 September 2014; published 18 November 2014)
}

\begin{abstract}
We consider the orbital magnetic properties of noninteracting charge carriers in graphene-based nanostructures in the low-energy regime. The magnetic response of such systems results both from bulk contributions and from confinement effects that can be particularly strong in ballistic quantum dots. First we provide a comprehensive study of the magnetic susceptibility $\chi$ of bulk graphene in a magnetic field for the different regimes arising from the relative magnitudes of the energy scales involved, i.e., temperature, Landau-level spacing, and chemical potential. We show that for finite temperature or chemical potential, $\chi$ is not divergent although the diamagnetic contribution $\chi_{0}$ from the filled valance band exhibits the well-known $-B^{-1 / 2}$ dependence. We further derive oscillatory modulations of $\chi$, corresponding to de Haas-van Alphen oscillations of conventional two-dimensional electron gases. These oscillations can be large in graphene, thereby compensating the diamagnetic contribution $\chi_{0}$ and yielding a net paramagnetic susceptibility for certain energy and magnetic field regimes. Second, we predict and analyze corresponding strong, confinement-induced susceptibility oscillations in graphene-based quantum dots with amplitudes distinctly exceeding the corresponding bulk susceptibility. Within a semiclassical approach we derive generic expressions for orbital magnetism of graphene quantum dots with regular classical dynamics. Graphene-specific features can be traced back to pseudospin interference along the underlying periodic orbits. We demonstrate the quality of the semiclassical approximation by comparison with quantum-mechanical results for two exemplary mesoscopic systems, a graphene disk with infinite mass-type edges, and a rectangular graphene structure with armchair and zigzag edges, using numerical tight-binding calculations in the latter case.
\end{abstract}

DOI: 10.1103/PhysRevB.90.205424

PACS number(s): 73.22.Pr, 73.20.At, 03.65.Sq, 75.20.-g

\section{INTRODUCTION}

Since the seminal work of Landau [1] it is known that a conventional free electron gas exhibits a weak diamagnetic orbital magnetic response. In two dimensions and at low magnetic field, its magnetic susceptibility $\chi$ is just a constant, i.e., independent of Fermi energy and $B$ field. For Dirac fermions in two dimensions, e.g., charge carriers in graphene close to the charge neutrality point, the situation is different: As McClure showed nearly 50 years ago [2], a noninteracting two-dimensional (2D) system of massless Dirac fermions features a Curie-type $1 / k_{\mathrm{B}} T$ behavior [3] at finite temperature $T$ that merges, for vanishing temperature, into a peculiar dependence on the chemical potential [2,4-11]: $\chi \sim \delta(\mu)$, i.e., a magnetic response that is divergent in the undoped limit and otherwise zero.

In this work we pose the question of how orbital magnetism in graphene-based nano- and mesoscale systems is altered through the presence of the confinement. Similar questions had been intensively discussed in the early 1990s for small disordered metallic rings [12], quasiballistic micron-sized rings [13], and square cavities [14] based on conventional 2D electron systems. The magnetic response of (ensembles of) these mesoscopic systems, namely the observed persistent current in the rings and the susceptibility of the cavities, turned out to exceed the bulk Landau diamagnetism by one to two orders of magnitude. These original experimental findings triggered broad theoretical activities (for reviews see [15-17]) investigating in particular also the role of noninteracting versus interacting contributions to the orbital magnetism. While twenty years ago further progress in the field had been hindered by experimental limitations, recent new high-precision cantilever magnetization (persistent current) measurements of ensembles of rings proved [18] the feasibility to reliably measure orbital magnetism of nanoscale objects. The results of these recent experiments are essentially in line with earlier theory based on noninteracting systems [19]. Given the peculiar orbital magnetic behavior of bulk graphene, and in view of the above-mentioned possibility to observe confinement-enhanced magnetism in nanostructures [18], it hence is of interest to explore also orbital magnetism in graphene nanostructures, a topic that has been barely addressed in the literature.

Here, we employ a trajectory-based semiclassical pathintegral formalism to compute the orbital magnetic susceptibility. As recently shown, such an approach is suitable for the quantitative description and interpretation of the density of states [20] and conductance [21] of graphenebased cavities. This approach allows for the incorporation of graphene-specific boundary effects (zigzag, armchair, and infinite mass). The confinement geometry and the type of edge is then encoded in the amplitudes and phases of paths (hitting the boundaries) that enter into the respective semiclassical trace formulas. We combine this approach with an earlier semiclassical treatment of orbital magnetism in conventional ballistic electron cavities [16,22]. We show that the susceptibility of graphene cavities of linear system size $R$ exhibits confinement-induced oscillations in $k_{\mathrm{F}} R$ where $k_{\mathrm{F}}$ is the Fermi momentum. For integrable geometries and at low temperatures their amplitude is parametrically larger by a factor of $\sqrt{k_{\mathrm{F}} R}$ than the corresponding bulk susceptibility. However, graphene cavities additionally carry features of bulk graphene. Hence, in the first part of the paper we include a comprehensive discussion of graphene bulk orbital magnetism. While a number of previous works addressed various parameter regimes separately we aim at a systematic presentation of the various bulk regimes. 


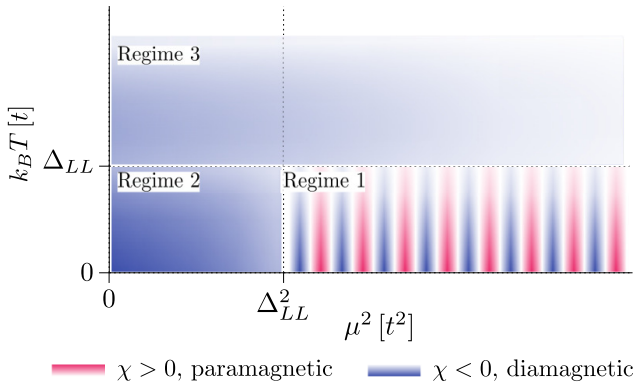

FIG. 1. (Color online) Rough schematic overview of the orbital magnetic behavior of graphene in a perpendicular field for the energy regimes studied in Sec. IIID. Here $\mu$ is the chemical potential, $k_{B} T$ is the thermal energy and $\Delta_{L L} \propto \sqrt{B}$ represents the Landau-level spacing. Blue (red) regions refer to parameter regimes where graphene shows diamagnetism (paramagnetism). The color intensity roughly indicates the strength of the magnetic response. The magnetic susceptibility $\chi$ is diamagnetic in almost all areas except the de Haas-van Alphen regime, $\mu>\Delta_{L L}>k_{B} T$, with susceptibility oscillations of $\chi$ being linear in $\mu^{2}$ (and $1 / B$ ).

This is simplistically sketched in Fig. 1. It shows an overall diamagnetic behavior up to the energy region governed by de Haas-van Alphen oscillations [23-27] for $k_{\mathrm{B}} T<\Delta_{\mathrm{LL}}<\mu$, with $\Delta_{\text {LL }}$ proportional to the Landau-level spacing. However, the diamagnetic regions exhibit interesting parametrical dependencies that we will derive and review. For instance, the afore-mentioned divergent behavior of $\chi$ at $T=0$ is smoothed out if $k_{\mathrm{B}} T$ is bigger than the mean level spacing.

The paper is organized as follows: After summarizing the necessary thermodynamic formalism in Sec. II, we first give a comprehensive account of bulk magnetism in graphene in Sec. III, addressing the various parameter regimes mentioned above. This also involves introducing our numerical approach and our scheme to extract bulk results from the numerics performed for finite systems. In the other main Sec. IV we consider in detail finite-size effects in the orbital magnetic response of nanostructued graphene. There we generalize the existing semiclassical approaches to quantitatively describe and interpret oscillatory effects in the susceptibility. These semiclassical predictions are compared to corresponding quantum calculations for disklike and rectangular geometries. We focus on integrable structures since chaotic or diffusive geometries are expected to exhibit a parametrically weaker magnetic response.

\section{BASIC THERMODYNAMIC QUANTITIES}

In order to investigate the orbital magnetic properties of a quasi-two-dimensional solid in general, it is convenient to start from the total grand potential in the presence of a perpendicular magnetic field of strength $B$,

$$
\Omega(\mu, B)=-\frac{1}{\beta} \int_{-\infty}^{\infty} d E \rho(E, B) \ln \left[1+e^{-\beta(E-\mu)}\right],
$$

where $1 / \beta=k_{\mathrm{B}} T$ denotes the thermal energy. The chemical potential $\mu$ is assumed to be $B$ independent. The total density of states [28] (DOS), $\rho(E, B)=\rho_{v}(E, B)+\rho_{c}(E, B)$, comprises conduction- and valence-band states simultaneously as well as the field dependence of the energy spectrum of the solid. Defining $E_{v / c}$ as the energy of the band edge of the valence/conduction band, the corresponding densities of states fulfill $\rho_{v / c}(E, B)=0, \forall E \gtrless E_{v / c}$, even for a vanishing energy gap $E_{g}=E_{c}-E_{v}=0$ as in the case of graphene. Without loss of generality, $\mu$ is chosen to be larger than $E_{v}$. Due to the properties of the total DOS, the grand potential can be decomposed as $\Omega=\Omega_{v}+\Omega_{c}$, where

$$
\begin{aligned}
& \Omega_{v}(\mu, B)=-\frac{1}{\beta} \int_{-\infty}^{E_{v}} d E \rho_{v}(E, B) \ln \left[1+e^{-\beta(E-\mu)}\right], \\
& \Omega_{c}(\mu, B)=-\frac{1}{\beta} \int_{E_{c}}^{\infty} d E \rho_{c}(E, B) \ln \left[1+e^{-\beta(E-\mu)}\right] .
\end{aligned}
$$

Equation (3) contains the contribution to $\Omega$ from electrons in the conduction band for Fermi energies $\mu>E_{v}$ or thermal excitation. In the limit $T \rightarrow 0$ only states with energy $E_{c} \leqslant$ $E \leqslant \mu$ are occupied. In view of

$$
-\lim _{\beta \rightarrow \infty} \frac{1}{\beta} \ln \left(1+e^{-\beta x}\right)=x \theta(-x),
$$

and taking the limit $T \rightarrow 0$ in Eq. (2), the contribution to $\Omega$ from the completely filled valence band is

$$
\Omega_{0}(\mu, B)=\int_{-\infty}^{E_{v}} d E \rho_{v}(E, B)(E-\mu) .
$$

In general, the integral (5) can diverge, if the particular model assumes a valence band without lower boundary. As we will discuss in Sec. IIIC for bulk graphene in the low-energy approximation, $\Omega_{0}$ can be decomposed into a $B$-field-dependent and a divergent part, which does not include any field dependence and therefore has no effect on the magnetic properties.

By pulling a factor exp $[-\beta(E-\mu)]$ out of the logarithm in Eq. (2) $\Omega_{v}$ can be represented as

$$
\Omega_{v}(\mu, B)=\Omega_{0}(\mu, B)-\frac{1}{\beta} \int_{-\infty}^{E_{v}} d E \rho_{v}(E, B) \ln \left[1+e^{\beta(E-\mu)}\right] .
$$

The second term in Eq. (6) contains a similar contribution to $\Omega$ as $\Omega_{c}$ corresponding to electron vacancies at finite temperature. As a first conclusion, $\Omega$ can be decomposed into the $T$-independent part $\Omega_{0}$, coming from the filled part of the valence band, and a contribution

$$
\begin{aligned}
\Omega_{T}(\mu, B)= & \Omega(\mu, B)-\Omega_{0}(\mu, B) \\
= & -\frac{1}{\beta} \int_{-\infty}^{\infty} d E\left\{\rho_{v}(E, B) \ln \left[1+e^{\beta(E-\mu)}\right]\right. \\
& \left.+\rho_{c}(E, B) \ln \left[1+e^{-\beta(E-\mu)}\right]\right\}
\end{aligned}
$$

due to excited electrons in the conduction band and holes in the valence band. Within the relevant temperature range the integral (8) converges fast due to the exponential decay of the integrand at both integration limits.

The total magnetic susceptibility is defined as

$$
\chi(\mu, B)=-\frac{\mu_{0}}{\mathcal{A}}\left(\frac{\partial^{2} \Omega(\mu, B)}{\partial B^{2}}\right)_{T, \mu} .
$$


In view of Eq. (7), it can be decomposed into

$$
\chi(\mu, B)=\chi_{0}(\mu, B)+\chi_{T}(\mu, B),
$$

with

$$
\chi_{x}(\mu, B)=-\frac{\mu_{0}}{\mathcal{A}}\left(\frac{\partial^{2} \Omega_{x}(\mu, B)}{\partial B^{2}}\right)_{T, \mu}, \quad x=0, T .
$$

Here, $\mathcal{A}$ denotes the area of the system and $\mu_{0}$ is the vacuum permeability. As will be shown in Sec. III C for bulk graphene, $\chi_{0}$, which is of similar origin as the Landau susceptibility $[1,16]$ of nonrelativistic electron gases, represents a smooth, diamagnetic contribution $\propto 1 / \sqrt{B}[3,5,11,26,29]$ to the total susceptibility. Contrarily, $\chi_{T}$ in Eq. (10) can yield an oscillatory contribution to $\chi$ for certain energy regimes. In bulk systems this oscillatory behavior refers to the de Haas-van Alphen effect $[23,30]$, whereas in finite systems additional modulations in $\chi$ occur as signatures of the confinement, see Sec. IV.

\section{BULK ORBITAL SUSCEPTIBILITY}

\section{A. Spectral properties of Landau quantized charge carriers with linear dispersion}

In this section the orbital magnetic properties of bulk graphene in the energy range of linear dispersion are discussed. The graphene sheet is assumed to lie in the $x-y$ plane perpendicular to an external, homogeneous $B$ field. Then the energies of the charge carriers are Landau quantized [1]. The Landau levels of massless Dirac-Weyl particles in two dimensions describing bulk graphene read [31-33]

$$
E_{n}=\operatorname{sgn}(n) \frac{\sqrt{2} \hbar v_{F}}{l_{B}} \sqrt{|n|},
$$

with $n \in \mathbb{Z}$. Here, $l_{B}=\sqrt{\phi_{0} /(2 \pi B)}$ denotes the magnetic length with the magnetic-flux quantum $\phi_{0}=h / e$. Every Landau level $E_{n}$ has a twofold spin degeneracy $g_{s}$ and valley degeneracy $g_{v}$ as well as a $\varphi=\phi / \phi_{0}$-fold degeneracy $(\phi=B \mathcal{A})$ which can be, e.g., deduced from phase-space arguments [23,34] and Bohr-Sommerfeld quantization [35] of the corresponding cyclotron orbits. Thus the orbital degeneracy in graphene is identical to that of Landau levels of ordinary 2D electron gases [1], $\epsilon_{n}=\left(\hbar / m^{*}\right) e B(n+1 / 2)$, with effective mass $m^{*}$ and $n \in \mathbb{N}_{0}$. In this case the lowest Landau level has the finite value $\epsilon_{0}=\left(\hbar / m^{*}\right) e B / 2$ while for graphene $E_{0}=0$ attains zero and lies precisely at the touching point of the conduction and valence bands. In the presence of a magnetic field conduction- and valence-band states occupy the zeroth Landau level equally leading to an increase of the total energy of the filled valence band. Thus the contribution $\chi_{0}$ from the filled valence band is expected to be diamagnetic as discussed in detail in Sec. III C. Whether the total susceptibility $\chi$, Eqs. (9) and (10), is para- or diamagnetic depends on the contribution $\chi_{T}$ of excited electrons and holes in the particular energy regime.

The single-particle DOS of bulk graphene,

$$
\rho(E, B)=g \varphi \sum_{n=-\infty}^{\infty} \delta\left[E-E_{n}(B)\right],
$$

can be decomposed into a smooth and an oscillatory part with respect to $E$ and $B$. By means of Poisson summation [36] of the Landau index $n$ one obtains

$$
\begin{aligned}
\rho(E, B) & =C|E|\left\{1+2 \sum_{m=1}^{\infty} \cos \left[\pi m\left(\frac{E l_{B}}{\hbar v_{F}}\right)^{2}\right]\right\} \\
& =\bar{\rho}(E)+\rho^{\mathrm{osc}}(E, B)
\end{aligned}
$$

with $C=g \mathcal{A} /\left[2 \pi\left(\hbar v_{F}\right)^{2}\right]$ and $g=g_{s} g_{v}$. Note that each term in Eqs. (14) and (15) and thereby the total DOS reflects particle-hole symmetry, i.e., $\rho(E, B)=\rho(-E, B)$, due to the nearest-neighbor hopping approximation underlying the effective Dirac Hamiltonian. The smooth part $\bar{\rho}(E)=C|E|$ is $B$-independent and identical to the bulk DOS of the field free system [37]. Hence the entire contribution to $\chi$ arises from the oscillatory part $\rho^{\text {osc }}(E, B)$ that can be rewritten as

$$
\begin{aligned}
\rho^{\mathrm{osc}}(E, B)= & g \varphi \sum_{n=1}^{\infty}\left\{\delta\left[E-E_{n}(B)\right]+\delta\left[E+E_{n}(B)\right]\right\} \\
& +g \varphi \delta(E)-C|E| .
\end{aligned}
$$

This representation clearly indicates that the orbital magnetism arises only from Landau levels with $n \neq 0$. The zeroth Landau level leads to a $\varphi$-linear contribution to $\Omega$ and thus does not contribute to $\chi$. As for the DOS the related thermodynamic potentials can be decomposed into

$$
\Omega(\mu, B)=\bar{\Omega}(\mu)+\tilde{\Omega}(\mu, B) .
$$

Each term in Eq. (17) can be further split as shown in Eqs. (7) and (10), i.e., $X=X_{0}+X_{T}$, where $X=\bar{\Omega}, \tilde{\Omega}$. Note that $\bar{\Omega}$ arises directly from the field independent bulk DOS $\bar{\rho}(E)$, and hence $\chi \propto \partial^{2} \Omega /\left(\partial B^{2}\right)=\partial^{2} \tilde{\Omega} /\left(\partial B^{2}\right)$. We will show below that though $\tilde{\Omega}$ arises from the oscillatory part of the DOS, it yields not only an oscillatory but also a smooth contribution to the susceptibility.

\section{B. Comparability of numerical results with analytical bulk DOS calculations}

The comparison between the analytical results for bulk graphene, to be discussed in Secs. III C and III D, with the numerical tight-binding data of confined graphene quantum dots will demonstrate the importance of bulk effects in finite structures. Moreover, vice versa, we will employ the numerical calculations, restricted to finite geometries, to confirm the results from the effective bulk theory based on the Dirac equation. For such a comparison we need to extract the bulk contribution from the numerical results in an appropriate way as discussed below.

The finite systems considered have an equilateral triangular geometry with either pure armchair or zigzag boundaries. This particular choice of geometry enables also a distinct analysis of edge effects due to zigzag boundaries. Each system has mesoscopic dimensions, i.e., the triangle side lengths are $\mathcal{L} \approx 100 a$, where $a$ is the graphene lattice constant, such that the region of linear dispersion contains enough energy levels to require good comparability with the theory. The eigenenergies of the triangles are calculated within tight-binding approximation [38,39] including only nearest-neighbor hopping $t$ and using the Lanczos algorithm [40,41]. Figure 2 shows the 

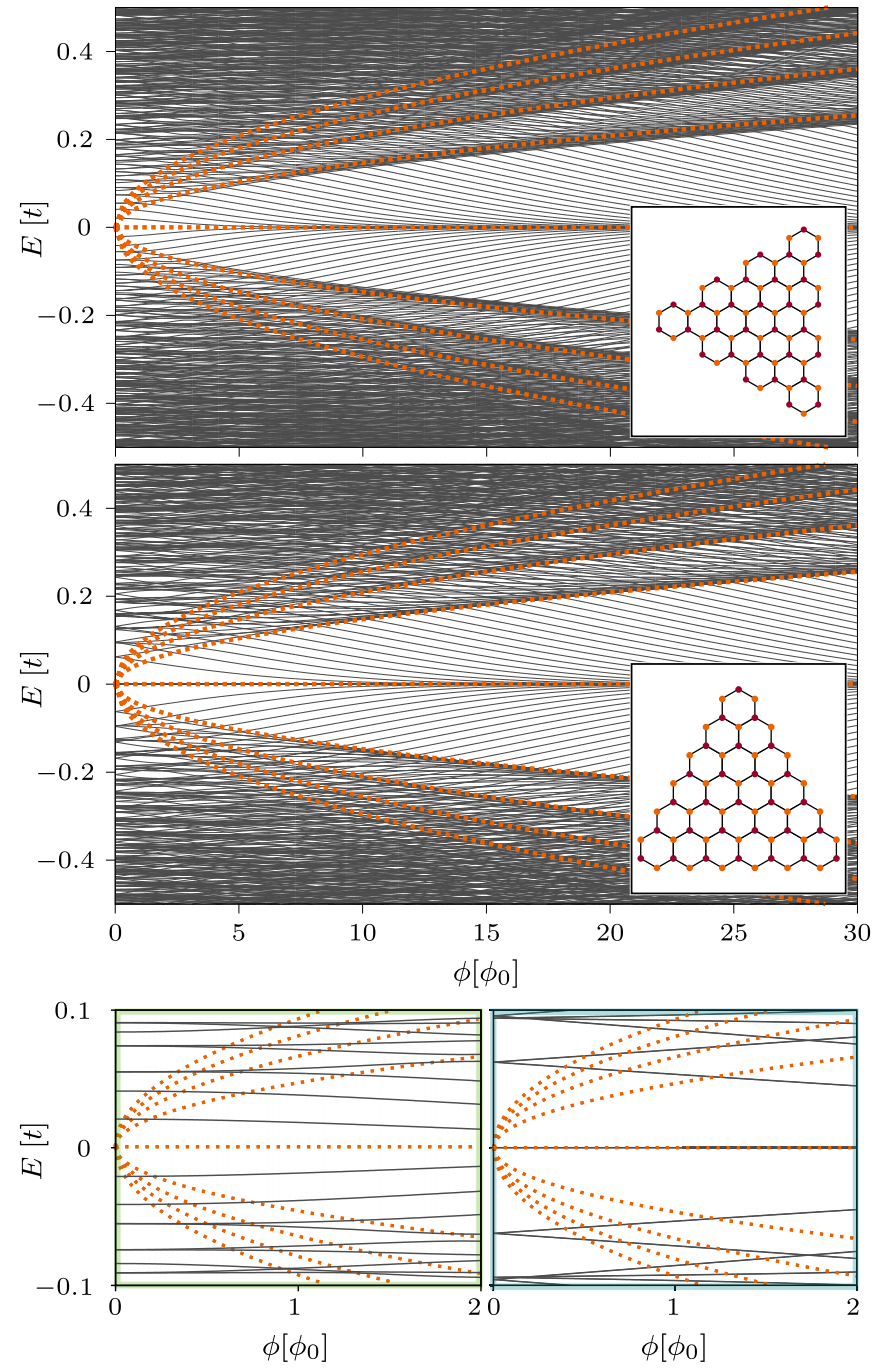

FIG. 2. (Color online) Energy spectrum of triangular quantum dots with armchair edge (upper panel) and zigzag edge (middle panel) as a function of the normalized magnetic flux through each system. The dashed orange lines refer to the lowest Landau energies $E_{n}$. In the case of zigzag geometry the zigzag edge states clearly appear close to $E \approx 0$ and contribute to the zeroth Landau level, as the enlarged section of the blue shaded region in the lower right panel shows. The lower left panel represents the green shaded region in the energy spectrum of the armchair triangular quantum dot, where no edge states occur. Insets: Sketch of the geometries; the actual systems considered are much larger: $\mathcal{L} \simeq 100 a$.

resulting energy spectrum for conduction- and valence-band energies $|E| \leqslant 0.55 t$ as a function of the normalized magnetic flux $\phi / \phi_{0}$. One can clearly see the condensation of the eigenenergies into Landau levels [42,43] for fluxes $\phi>5 \phi_{0}$. This is the regime where bulk effects should be distinctly observable in the finite systems.

In Subsec. III C we calculate the contribution $\chi_{0}$ from the valence band in Dirac approximation, which corresponds to the Landau susceptibility [1,16] of electron gases. Therefore we assume an unbounded valence band with linear dispersion which does not reflect the real band structure of graphene further away from the Dirac point. For this reason, $\chi_{0}$ is not accessible within tight-binding approximation even if one

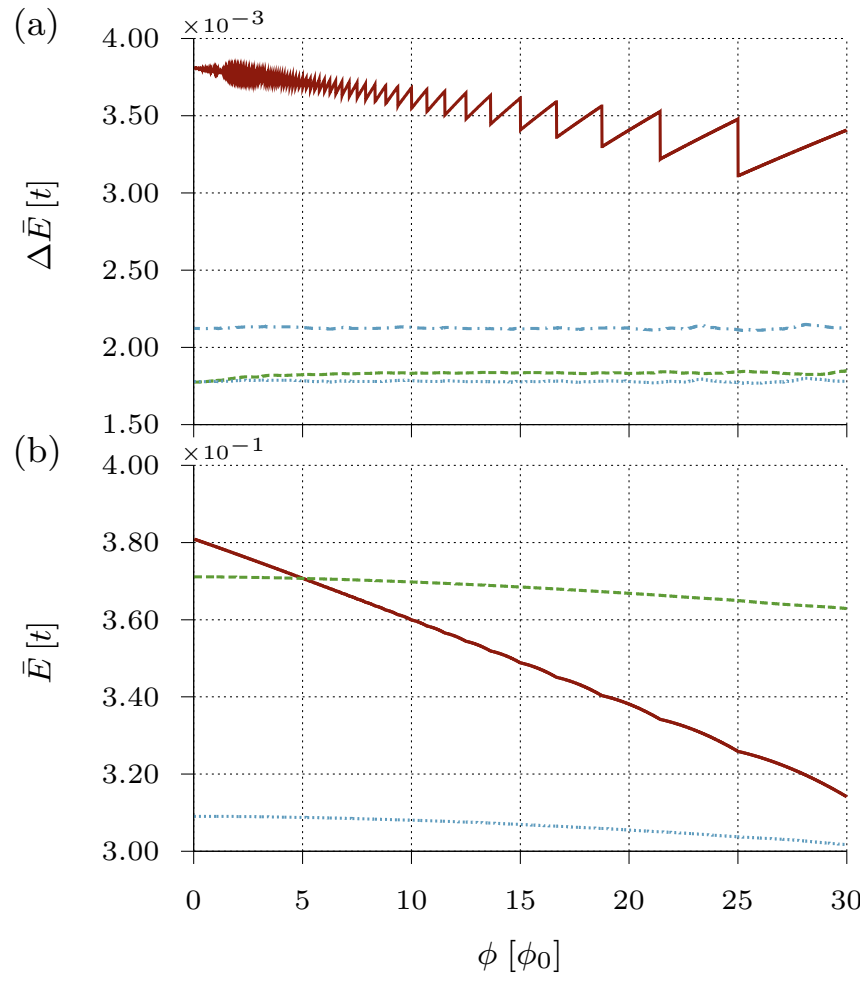

FIG. 3. (Color online) (a) Comparison of the average level spacing (in units of hopping energy $t$ ) of the lowest 600 electron states of the two triangular quantum dots, see Fig. 2, with the Dirac model for bulk graphene (red solid line) calculated from Eq. (12) as a function of the magnetic flux. The blue dotted (dashed-dotted) line represents the average level spacing of the zigzag system with (without) considering edge states. Due to the edge states $\Delta E$ is smaller than in the armchair system (green dashed line). (b) Mean energy of the lowest 600 electronic energies as a function of the magnetic flux. The full (red), dotted (blue), and dashed (green) lines correspond to the bulk, zigzag, and armchair system, respectively.

would go to very large system sizes. Thus the comparison of the analytic theory with numerical data for the quantum dots is restricted to the temperature-dependent part of $\tilde{\Omega}$ and $\chi$, respectively, where only the energy levels close to the Fermi level contribute.

As one can deduce from Fig. 2 the average level spacings $\Delta \bar{E}^{(a c, z z)}$ of the finite systems differ from the average Landaulevel spacing $\Delta \bar{E}^{\text {(bulk) }}$ of the bulk system. In Fig. 3(a) we compare explicitly $\Delta \bar{E}^{(x)}(x=a c, z z$, bulk $)$, calculated from the first $N=600$ electronic states for each case, as a function of the normalized magnetic flux, $\Delta \bar{E}^{(x)}(\phi)=1 /(N-$ 1) $\sum_{i=2}^{N} \Delta E_{i, i-1}^{(x)}(\phi)$, where $\Delta E_{i, i-1}^{(x)}=E_{i}^{(x)}-E_{i-1}^{(x)}>0$. The differences between $\Delta \bar{E}^{\text {(bulk) }}$ and $\Delta \bar{E}^{(a c, z z)}$ seen in Fig. 3(a) are caused by boundary effects which are encoded in the energy spectra of the quantum dots but not regarded within the analytic bulk calculations by definition. To consider them analytically in the case of graphene would require us to generalize calculations of nonoscillatory perimeter corrections to the bulk theory, as demonstrated in Refs. [44,45] for the example of Landau diamagnetism in electron gases.

When dealing with thermodynamic potentials and related observables, finite temperature $T$, encoded in the Fermi-Dirac 
TABLE I. Flux average of the mean energy $\left\langle\bar{E}^{(x)}\right\rangle_{\phi}$ and average level spacing $\left\langle\Delta \bar{E}^{(x)}\right\rangle_{\phi}$ for the first 600 electron states of bulk graphene, an armchair, and a zigzag triangular quantum dot, same as Fig. 2. The considered flux interval amounts to $\left[0,30 \phi_{0}\right]$. The number in parentheses comprises the edge states.

\begin{tabular}{lccc}
\hline \hline$x$ & Bulk & Armchair & Zigzag \\
\hline$\left\langle\Delta \bar{E}^{(x)}\right\rangle_{\phi}\left[10^{-3} t\right]$ & 3.514 & 1.828 & $2.124(1.780)$ \\
$\left\langle\bar{E}^{(x)}\right\rangle_{\phi}[t]$ & 0.349 & 0.368 & 0.306 \\
\hline \hline
\end{tabular}

statistics, implies an effective broadening $1 / \beta$ of each energy level as can be seen from Eq. (8). For an appropriate comparison between the Dirac-type bulk theory without considering boundary corrections and the tight-binding results for the finite-size structures the thermal energies chosen should obey

$$
\beta^{\text {(bulk) }} \Delta \bar{E}^{(\text {bulk })}(\phi) \approx \beta^{(x)} \Delta \bar{E}^{(x)}(\phi), x=a c, z z .
$$

To get reliable values from this expression the edge states are not considered in the case of the zigzag system since they lead to underestimating the average level spacing.

To compare the properties of the bulk system with those of the quantum dots for finite magnetic flux it is necessary to average Eq. (18) over the flux interval considered. The resulting level spacings averaged over $\phi \in\left[0,30 \phi_{0}\right]$ can be read off from Table I. The above procedure, providing an adequate comparison between all three systems, refers to the entire thermodynamic potentials and related properties. Independently, the individual energy levels for each of the considered systems can be written as $E_{i}^{(x)}=\bar{E}^{(x)}+\delta E_{i}^{(x)}$, where $\bar{E}^{(x)}=1 / N \sum_{i=1}^{N} E_{i}^{(x)}$ denotes the mean energy of the $N$ valence-band states considered. Figure 3(b) shows the flux dependence of $\bar{E}^{(x)}$ for each system averaged over the lowest $N=600$ electron states. In all three cases the mean energies are of the same order of magnitude. Due to the contribution of edge states, $\bar{E}^{a c}>\bar{E}^{z z}$.

The grand potential for each system reads

$$
\Omega^{(\mathrm{x})}(\mu, B)=-\frac{1}{\beta} \sum_{i} \ln \left[1+e^{-\beta\left(\bar{E}^{(x)}+\delta E_{i}^{(x)}-\mu\right)}\right] .
$$

The properties of the exponential function and the logarithm yield a rough scaling behavior of $\Omega^{(a c, z z)} / \Omega^{(\text {bulk })} \approx \gamma^{(a c, z z)}$ for each system reflecting in first approximation

$$
\beta^{a c, z z} \bar{E}^{(a c, z z)}-\beta^{\text {bulk }} \bar{E}^{\text {(bulk })} \gtrless 0 .
$$

Resulting differences in the absolute value of $\Omega$ and $\chi$, respectively, for fixed $\mu$ and $\varphi$ can be approximately compensated by rescaling the bulk value with the factor $\gamma^{(a c, z z)}$. The factors $\gamma^{(a c, z z)}$ were obtained by fitting using the LevenbergMarquardt [46] algorithm.

As a consequence of $\bar{E}^{a c}>\bar{E}^{z z}$, Fig. 3(b), and Eq. (19) we expect the susceptibility contribution $\chi_{T}$ for a zigzag triangular quantum dot to be smaller than for the corresponding armchair system at the same temperature corresponding to Eq. (20). This behavior is also confirmed in Ref. [47], where the orbital magnetic properties of hexagonal and triangular graphene nanostructures are numerically studied within tight-binding approximation.

\section{Susceptibility contribution from filled valence band}

As discussed in Sec. III A, the susceptibility contribution $\chi_{T}$ from the filled valence band, Eq. (5), can be evaluated from Eq. (8) by using only the field-dependent part of the DOS, $\rho^{\text {osc }}(E, B)$ :

$$
\begin{aligned}
\tilde{\Omega}_{0}(\mu, B)= & \int_{-\infty}^{0} d E \rho^{\mathrm{osc}}(E, B)(E-\mu) \\
= & -2 C \sum_{m=1}^{\infty} \operatorname{Re}\left[\lim _{\eta \rightarrow 0} \int_{0}^{\infty} d E\left(E^{2}+\mu E\right)\right. \\
& \left.\times e^{-\left[\eta-i \pi m\left(l_{B} / \hbar v_{F}\right)^{2}\right] E^{2}}\right] .
\end{aligned}
$$

Solving this integral and taking the limit $\eta \rightarrow 0$ yields

$$
\tilde{\Omega}_{0}(B)=\frac{K}{2} \varphi^{3 / 2} \sum_{m=1}^{\infty} \frac{1}{m^{3 / 2}}=\frac{K}{2} \varphi^{3 / 2} \zeta\left(\frac{3}{2}\right),
$$

where all prefactors are absorbed in the constant,

$$
K=4 \sqrt{\pi} C\left(\frac{\hbar v_{F}}{\sqrt{\mathcal{A}}}\right)^{3}=2 g \frac{\hbar v_{F}}{\sqrt{\mathcal{A} \pi}} .
$$

Indeed, $\Omega_{0}$ and thereby the corresponding susceptibility

$$
\chi_{0}(B)=-\frac{\mu_{0} g}{\phi_{0}^{2}} \hbar v_{F} \sqrt{\frac{\mathcal{A}}{\pi}} \frac{3 \zeta\left(\frac{3}{2}\right)}{4} \frac{1}{\sqrt{\varphi}} \propto-\frac{1}{\sqrt{B}}
$$

are independent of the chemical potential. $\chi_{0}(B)$ is diamagnetic because the grand potential of the valence band, $\bar{\Omega}_{0}+$ $\tilde{\Omega}_{0}(B)$, increases in the presence of a perpendicular magnetic field, i.e., $\tilde{\Omega}_{0}(B)>\tilde{\Omega}_{0}(0)$. The susceptibility $\chi_{0}$ diverges as $1 / \sqrt{B}$ implying that small variations of the flux cause huge changes in the magnetization of bulk graphene in the low-field regime. The scaling behavior (25) of $\chi_{0}$ was first discovered by McClure in 1956 within his studies of the diamagnetic properties of graphite [2] and confirmed by various research groups $[3,5,11,26,29]$ for monolayer graphene. In Sec. III D we show that this singularity of $\chi_{0}$, however, need not lead to a divergence of the total susceptibility $\chi=\chi_{0}+\chi_{T}$.

In the case of a bulk 2D electron gas (2DEG) the quantity corresponding to $\chi_{0}$ is the Landau susceptibility [16]

$$
\chi_{L}=-\mu_{0} g_{s} \frac{\pi}{6} \frac{\hbar^{2}}{\phi_{0}^{2} m^{*}} .
$$

It is also independent of $\mu$ but moreover does not depend on $B$. To estimate the relative strength of graphene diamagnetism we consider the ratio $\chi_{0} / \chi_{L}$ which reads

$$
\frac{\chi_{0}(B)}{\chi_{L}} \approx 0.2 \frac{m^{*}}{m_{e^{-}}} \sqrt{\frac{\mathcal{A}}{\varphi}} \mathrm{nm}^{-1} .
$$

To give an explicit example, consider GaAs $\left(m^{*}=0.067 m_{e^{-}}\right)$ and a graphene flake with a typical length $\mathcal{L} / l_{B} \gg 1$, such that bulk effects dominate over finite-size signatures in $\chi$. Choosing typical values $\phi=\phi_{0}$ and $\mathcal{A} \approx 100^{2} \mathrm{~nm}^{2}$, Eq. (27) yields $\chi_{0} \approx \chi_{L}$, i.e., the diamagnetic contribution from the valence band in graphene is comparable to the Landau susceptibility of a 2DEG for a magnetic field of $B \approx 0.5 \mathrm{~T}$. 


\section{Susceptibility contribution from thermally excited charge carriers}

To investigate the contribution to $\chi$ from excited electrons and holes we start from Eq. (8) considering only the fielddependent part $\rho^{\text {osc }}(E, B)$ of the DOS in Eq. (14):

$$
\begin{aligned}
\tilde{\Omega}_{T}(\mu, B)= & -\frac{1}{\beta} \int_{-\infty}^{\infty} d E \rho^{\mathrm{osc}}(E, B)\left\{\theta(-E) \ln \left[1+e^{\beta(E-\mu)}\right]\right. \\
& \left.+\theta(E) \ln \left[1+e^{-\beta(E-\mu)}\right]\right\} .
\end{aligned}
$$

Due to the integration over energy and the temperature dependence of $\tilde{\Omega}_{T}$ the corresponding susceptibility $\chi_{T}$ can contain a smooth as well as an oscillatory part, which is directly accessible within the semiclassical description of finite-size contributions to $\chi$ as shown in Sec. IV.

For the following considerations it is useful to integrate Eq. (28) twice by parts yielding

$$
\tilde{\Omega}_{T}(\mu, B)=\int_{-\infty}^{\infty} d E \mathcal{N}(E, B) f^{\prime}(E-\mu),
$$

with the integral over particle number fluctuations,

$$
\begin{aligned}
\mathcal{N}(E, B) & =\int_{0}^{E} d E^{\prime} \int_{0}^{E^{\prime}} d E^{\prime \prime} \rho^{\mathrm{osc}}(E, B) \\
& =K \varphi^{3 / 2} \sum_{m=1}^{\infty} \frac{S\left(\sqrt{\pi m} \frac{|E| l_{B}}{\hbar v_{F}}\right)}{m^{3 / 2}} .
\end{aligned}
$$

Here, $S(x)=\sqrt{2 / \pi} \int_{0}^{x} d t \sin \left(t^{2}\right)$ is the Fresnel integral [48] and $K$ is defined by Eq. (24). In Eq. (29)

$$
f^{\prime}(x)=-\frac{\beta}{4} \operatorname{sech}^{2}\left(\frac{\beta}{2} x\right) \stackrel{\beta \rightarrow \infty}{\longrightarrow}-\delta(x)
$$

denotes the derivative of the Fermi distribution function $f(x)=[1+\exp (\beta x)]^{-1}$. We rewrite Eq. (29) as

$$
\tilde{\Omega}_{T}(\mu, B)=K \varphi^{3 / 2} \sum_{m=1}^{\infty} \frac{\omega_{m, T}(\mu, B)}{m^{3 / 2}},
$$

where $\omega_{m, T}$ is defined as the energy integral

$$
\omega_{m, T}(\mu, B)=\int_{-\infty}^{\infty} d E S\left(\sqrt{\pi m} \frac{|E| l_{B}}{\hbar v_{F}}\right) f^{\prime}(E-\mu) .
$$

Since the integral (34) cannot generally be solved analytically, it is convenient to discuss separately different regimes defined through the ratios between the relevant length scales entering the problem, namely the magnetic length $l_{B}$, the Fermi wavelength $\lambda_{F}=\hbar v_{F} / \mu$, and the thermal wavelength $\lambda_{T}=\hbar v_{F} \beta$. For the sake of simplicity we define the two dimensionless parameters

$$
\alpha=\frac{\lambda_{F}}{l_{B}} \propto \frac{\Delta_{L L}}{\mu}, \quad \gamma=\frac{\lambda_{T}}{l_{B}} \propto \frac{\Delta_{L L}}{k_{B} T},
$$

where $\Delta_{L L} \propto \sqrt{B}$ denotes the energy spacing between adjacent Landau levels.

\section{Regime: $\gamma>1>\alpha$}

In this parameter range the Landau-level spacing is larger than or comparable to the thermal energy, but smaller than the chemical potential. The resulting temperature-dependent contribution to $\Omega$ is therefore expected to show an oscillatory modulation as a function of $\mu$ or $\varphi$ known as the de Haas-van Alphen effect in electron gases [23-25]. Moreover we will show that the $1 / \sqrt{B}$ singularity in Eq. (25) is canceled. Hence it is useful to decompose the Fresnel integral in Eq. (34) into its smooth and oscillatory part, i.e., $\operatorname{sgn}(x) S(x)=1 / 2+\tilde{\mathrm{S}}(x)$. The function $\tilde{\mathrm{S}}(x)$ oscillates around zero and can be written in terms of the hypergeometric function $U\left(1 / 2 ; 1 / 2,-i x^{2}\right)$ or its integral representation as shown in Appendix A,

$$
\tilde{\mathrm{S}}(x)=-\frac{1}{\sqrt{2 \pi}} \operatorname{Im}\left[\int_{0}^{\infty} d u \frac{e^{-(u-i) x^{2}}}{\sqrt{\pi u}(u-i)}\right] .
$$

Then the energy integral (34) reads

$$
\omega_{m, T}=-\frac{1}{2}+\int_{-\infty}^{\infty} d E \tilde{\mathrm{S}}\left(\sqrt{\pi m} \frac{|E| l_{B}}{\hbar v_{F}}\right) f^{\prime}(E-\mu) .
$$

Note that the remaining integral directly leads to the $B$ fielddependent part of the total grand potential, $\tilde{\Omega}=\tilde{\Omega}_{0}+\tilde{\Omega}_{T}$, since the first term in Eq. (37) exactly cancels with $\tilde{\Omega}_{0}$, Eq. (28), after inserting it into Eq. (33). Then $\tilde{\Omega}$ can be cast into the form

$$
\tilde{\Omega}(\mu, B)=\sum_{m=1}^{\infty} \frac{K \varphi^{3 / 2}}{\sqrt{2 \pi m^{3}}} \operatorname{Im}\left[\int_{0}^{\infty} d u \frac{\mathrm{Y}_{T}(\mu, B, u)}{\sqrt{\pi u}(u-i)}\right],
$$

with

$$
\mathrm{Y}_{T}(\mu, B, u)=\int_{-\infty}^{\infty} d E f^{\prime}(E-\mu) e^{-(u-i) \pi m\left(\frac{E l_{B}}{\hbar v_{F}}\right)^{2}} .
$$

As shown in Appendix A of Ref. [16] for a similar situation,

$$
Y_{T}(\mu, B, u) \approx Y_{0}(\mu, B, u) R_{T}\left[\phi^{\prime}(\mu, B, u)\right],
$$

where the temperature damping factor $R_{T}$ is defined as

$$
R_{T}\left[\phi^{\prime}(\mu, B, u)\right]=\frac{\frac{\pi}{\beta} \phi^{\prime}(\mu, B, u)}{\sinh \left[\frac{\pi}{\beta} \phi^{\prime}(\mu, B, u)\right]} \stackrel{\beta \rightarrow \infty}{\longrightarrow} 1
$$

and results from the derivative of the Fermi-Dirac distribution and $\phi^{\prime}(\mu, B, u)=\partial \phi(E, B, u) /\left.(\partial E)\right|_{E=\mu}$. From Eqs. (40) and (41) follows

$$
Y_{T}(\mu, B, u) \approx e^{-(u-i) \pi m / \alpha^{2}} R_{T}\left(\beta \frac{2 \pi m}{\alpha \gamma}\right),
$$

so the field-dependent part of $\Omega$ finally reads

$$
\tilde{\Omega}(\mu, B) \approx K \sum_{m=1}^{\infty} \frac{\varphi^{3 / 2}}{m^{3 / 2}} \tilde{\mathrm{S}}\left(\frac{\sqrt{\pi m}}{\alpha}\right) R_{T}\left(\beta \frac{2 \pi m}{\alpha \gamma}\right) .
$$

Compared to the rapid magneto oscillations of $\tilde{\mathrm{S}}$, the factor $R_{T}$ only slowly varies on the relevant scales so that its magnetic field derivatives can be neglected in the calculation of the total magnetic susceptibility:

$$
\begin{aligned}
\chi(\mu, B) & =-\frac{\mu_{0} g}{\phi_{0}^{2}} \hbar v_{F} \frac{3 \sqrt{\mathcal{A}}}{2 \pi} \sum_{m=1}^{\infty} \frac{R_{T}\left(\beta \frac{2 \pi m}{\alpha \gamma}\right)}{m^{3 / 2}} \frac{J\left(\frac{\sqrt{\pi m}}{\alpha}\right)}{\sqrt{\varphi}} \\
& =\chi_{0}(B) \times \frac{2}{\sqrt{\pi} \zeta\left(\frac{3}{2}\right)} \sum_{m=1}^{\infty} \frac{R_{T}\left(\beta \frac{2 \pi m}{\alpha \gamma}\right)}{m^{3 / 2}} J\left(\frac{\sqrt{\pi m}}{\alpha}\right),
\end{aligned}
$$


with $\chi_{0}(B)$ defined in Eq. (25). At finite temperatures the sum in Eq. (45) is exponentially damped due to $R_{T}$, ensuring convergence of the corresponding expression. The function $J(x)$ is defined as

$$
J(x)=\tilde{\mathbf{S}}(x)+\sqrt{\frac{2}{\pi}} x\left[\sin \left(x^{2}\right)-\frac{2 x^{2}}{3} \cos \left(x^{2}\right)\right],
$$

yielding $\mu^{2}$ - as well as $1 / \phi$-periodic oscillations of $\chi$, respectively $\chi_{T}=\chi-\chi_{0}$, which can be extracted from Eqs. (45) and (25). This becomes more obvious by transforming the expression (46) for $J(x)$ into

$$
\begin{aligned}
J(x)= & -\frac{\cos \left(x^{2}\right)}{\sqrt{2 \pi}}\left[\Sigma_{1}\left(x^{2}\right)+\frac{4}{3} x^{3}\right] \\
& -\frac{\sin \left(x^{2}\right)}{\sqrt{2 \pi}}\left[\Sigma_{2}\left(x^{2}\right)-2 x\right]
\end{aligned}
$$

by defining $\Sigma_{1 / 2}\left(x^{2}\right)=\operatorname{Im} / \operatorname{Re}\left[\exp (i \pi / 4) U\left(1 / 2 ; 1 / 2 ;-i x^{2}\right)\right]$ and rewriting $\tilde{\mathrm{S}}(x)$, Eq. (36). For the magnetization of bulk graphene an expression similar to Eq. (45) is derived in Ref. [26] considering additionally a band gap and impurity scattering, whereas in Ref. [27] the effect of an additional in-plane electric field is studied.

In Fig. 4 the oscillatory behavior of $\chi_{T}$ is demonstrated. In panel (a) $\chi_{T}$ exhibits equidistant extrema when plotted as a function of $\mu^{2}$ at $\phi=15 \phi_{0}$. Panel (b) shows the $1 / \phi$ periodicity of $\chi_{T}$ at $\mu=0.3 t$. In both cases the thermal energy is chosen such that $1 / \beta^{\text {(bulk) }} \approx 3 \times 10^{-3} t$. The amplitude of the $\chi_{T}$ oscillations is about one order of magnitude larger than $\left|\chi_{0}\right|$, implying that the full orbital susceptibility $\chi$ of graphene oscillates between strong diamagnetic but also paramagnetic behavior as a function of $\mu$ and $B$, respectively.

In Fig. 5(a) we show the numerically calculated susceptibility contribution $\chi_{T}$ for a triangular armchair and zigzag quantum dot for the same value of the magnetic flux as in Fig. 4(a), i.e., $\phi=15 \phi_{0}$. The thermal energies are chosen as $1 / \beta^{(a c, z z)} \approx 1 \times 10^{-3} t$ to satisfy relation (18). The levels of the finite systems are then well resolved leading to extra peaks with smaller amplitude in between those caused by

(a)

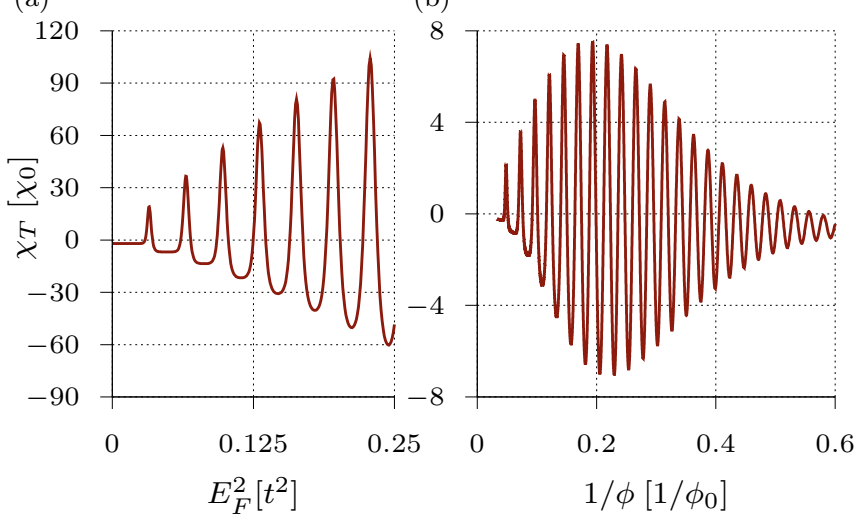

FIG. 4. (Color online) Susceptibility contribution $\chi_{T}$ for bulk graphene calculated from Eqs. (44) and (45) at $1 / \beta^{\text {(bulk) }} \approx 3 \times 10^{-3} t$. (a) $\chi_{T}$ shown as a function of $\mu^{2}$ for fixed $\phi=15 \phi_{0}$. (b) $\chi_{T}$ plotted as a function of the inverse magnetic flux for fixed $\mu=0.3 t$.

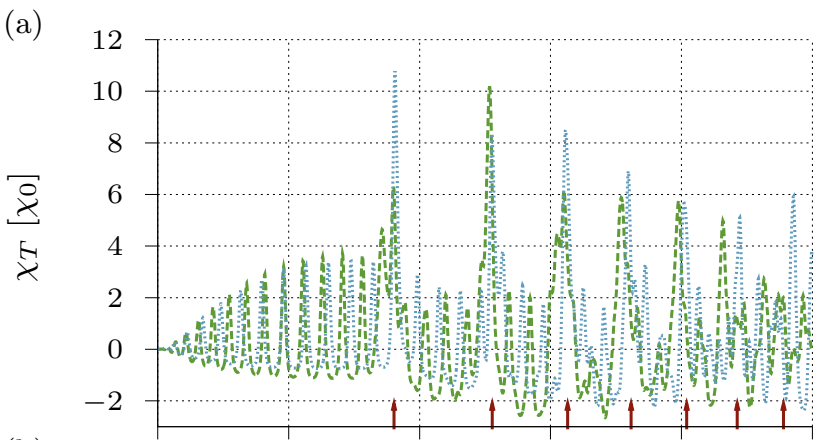

(b)

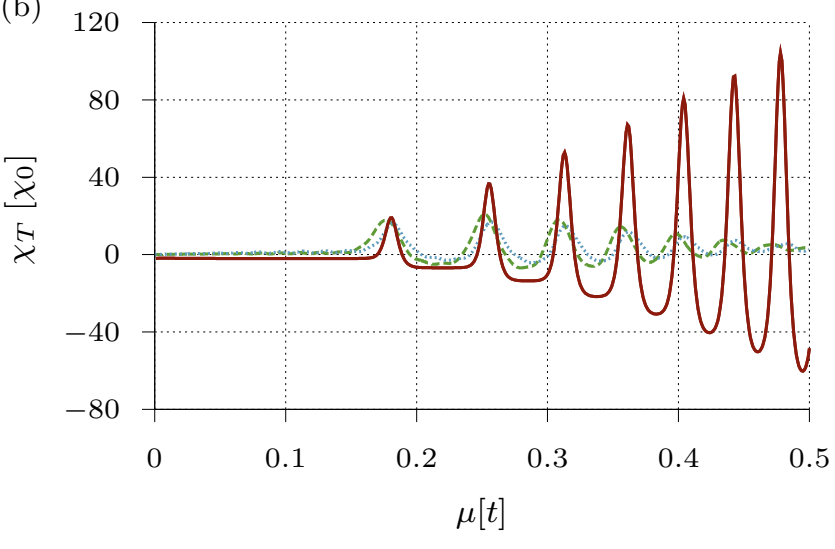

bulk — ac -------.. $\mathrm{ZZ}$

FIG. 5. (Color online) Oscillatory susceptibility contribution $\chi_{T}$ for triangular graphene cavities as a function of the chemical potential at $\phi=15 \phi_{0}$. (a) $\chi_{T}$ for armchair (green dashed) and zigzag (blue dotted) confinement with side length $\mathcal{L} \approx 100 a$ at $1 / \beta^{(a c, z z)} \approx 10^{-3} t$. The red arrows indicate the peak positions in the case of bulk graphene where only Landau levels exist. (b) Comparison of $\chi_{T}$ of the finite systems (dashed and dotted) at a slightly higher thermal energy $1 / \beta^{(a c, z z)}=5 \times 10^{-3} t$ with the corresponding bulk result (solid) at $1 / \beta^{\text {(bulk) }} \approx 3 \times 10^{-3}$.

level clustering in the vicinity of Landau levels (see Fig. 2). The latter are indicated by red arrows in Fig. 5(a) and coincide with the maxima in $\chi_{T}$ of the bulk system. These extra peaks are signatures of the confinement of the system and not captured within the bulk theory. Similar signatures are numerically observed in Ref. [47] for triangular but also hexagonal graphene quantum dots. In Sec. IV we will show how one can interpret these finite-size signatures within a semiclassical approach using periodic orbit theory. The amplitudes of the susceptibility oscillations of the quantum dots exceed the contribution $\chi_{0}$ from the filled valence band as well, implying that for certain ranges of $\phi$ and $\mu$ the total orbital magnetic susceptibility can become paramagnetic. By raising the thermal energy to $1 / \beta^{(a c, z z)} \approx 5 \times 10^{-3} t$ the finite-size features are smeared out and only extrema at the positions of the Landau levels survive as Fig. 5(b) demonstrates.

Figure 6 compares the susceptibility contribution $\chi_{T}$ of the triangular quantum dots with the bulk system as a function of $\phi$ at $\mu=0.3 t$ and $1 / \beta^{\text {(bulk) }} \approx 3 \times 10^{-3} t$, respectively $1 / \beta^{(a c, z z)}=5 \times 10^{-3} t$, such that finite-size effects are smeared out. For flux values $\phi \gtrsim 10 \phi_{0}$ the peak positions coincide very well. This corresponds to the spectral regime 


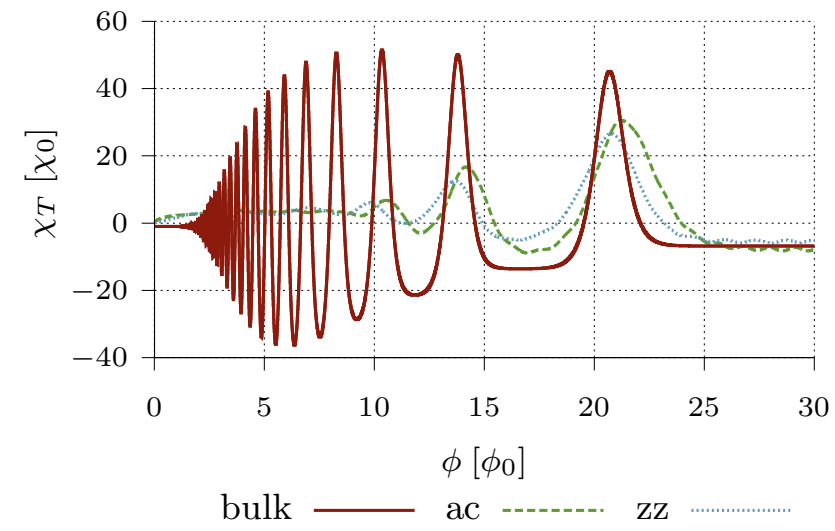

FIG. 6. (Color online) Susceptibility contribution $\chi_{T}$ as a function of $\phi$ (for same triangular quantum dots as in Fig. 5) for $\mu=$ $0.3 t, 1 / \beta^{\text {(bulk) }} \approx 3 \times 10^{-3} t$ and $1 / \beta^{(a c, z z)}=5 \times 10^{-3} t$. The maxima coincide well with the bulk case for $\phi \gtrsim 10 \phi_{0}$, i.e., the flux range where the bulk theory is applicable to the spectra of the finite quantum dots (see Fig. 2).

of the finite systems (see Fig. 2) where the levels cluster in the vicinity of Landau levels and the influence of the boundaries becomes negligible.

\section{Regime: $\alpha, \gamma>1$}

When the thermal energy and chemical potential are comparable to or smaller than the Landau-level spacing the temperature-dependent part of the susceptibility is expected to vanish [3]. If the magnetic field is tuned to very high values such that $\alpha, \gamma \gg 1$ the degeneracy of each Landau level rises accordingly, and all occupied states condense into the first or even to the zeroth level $E_{0}$. This yields a contribution to the temperature-dependent part of the total grand potential $\Omega_{T}$ linear in $B$ as mentioned in Sec. III A. In order to calculate $\chi_{T}$ from Eq. (28) it is useful to apply the representation (16) for $\rho^{\text {osc }}$. Then it is sufficient to consider only the sum over the Landau indices since the other terms do not contribute to $\chi$. To this end we write

$\tilde{\Omega}_{T}(\mu, B)=\hat{\Omega}_{T}(\mu, B)-g \frac{\varphi}{\beta} \sum_{s= \pm 1} \sum_{n=1}^{\infty} \ln \left(1+e^{-\sqrt{2 n} \gamma+s(\gamma / \alpha)}\right)$,

where the $B$-linear term

$$
\hat{\Omega}_{T}(\mu, B)=-\frac{g}{2} \frac{\varphi}{\beta} \sum_{s= \pm 1} \ln \left(1+e^{s(\gamma / \alpha)}\right)-\bar{\Omega}_{T}(\mu)
$$

does not contribute to $\chi_{T}$. $\bar{\Omega}_{T}$, defined through Eqs. (7) and (15), is only based on the average DOS $\bar{\rho}=C|E|$. In order to get an appropriate expression for $\tilde{\Omega}_{T}$ in this parameter range we Taylor expand the logarithm and the exponential function in Eq. (48) using the condition $\gamma>1$. Resumming the resulting triple infinite sums yields

$$
\tilde{\Omega}_{T}(\mu, B) \approx \hat{\Omega}_{T}(\mu, B)-g \frac{\varphi}{\beta} \sum_{s \pm 1} \ln \left(1+e^{-\sqrt{2} \gamma+s(\gamma / \alpha)}\right),
$$

as shown in Appendix B. Only the second term contributes to $\chi$. It is identical to the contribution from the first electron- and holelike Landau level to $\tilde{\Omega}_{T}$ as a comparison with Eq. (48) shows. The susceptibility contribution from Eq. (50) then yields

$$
\begin{aligned}
\chi_{T}(\mu, B) & =-\frac{1}{8} \sqrt{\frac{\pi}{2}} \frac{\mu_{0} g}{\phi_{0}^{2}} \hbar v_{F} \sqrt{\frac{\mathcal{A}}{\varphi}} \times F(\alpha, \gamma) \\
& =\chi_{0}(B) \times \frac{\pi}{6 \sqrt{2} \zeta\left(\frac{3}{2}\right)} \times F(\alpha, \gamma) .
\end{aligned}
$$

Here

$$
\begin{aligned}
F(\alpha, \gamma)= & \sum_{s= \pm 1}\left[3\left(1+e^{-\sqrt{2} \gamma+s(\gamma / \alpha)}\right)-\sqrt{2} \gamma\right] \\
& \times \operatorname{sech}^{2}\left[\frac{1}{2}\left(\sqrt{2} \gamma-s \frac{\gamma}{\alpha}\right)\right]
\end{aligned}
$$

can assume positive or negative values hence yielding a diaor paramagnetic susceptibility contribution. For $\gamma \gtrsim 1$, i.e., the level spacing is comparable to the thermal energy, $F(\alpha, \gamma)$ takes positive values, and hence $\chi_{T}$ is diamagnetic. In Ref. [3] the same parameter regime is discussed for the special case $\mu=0$ but treated in a slightly different way obtaining a diamagnetic result for $\chi_{T}$ which decays as a function of $\gamma$. In the range of validity of Eqs. (51) and (52) $\left|\chi_{T}\right|$ is at most half as large as $\left|\chi_{0}\right|$ as the following considerations show: In its validity range, $F$ approaches a supremum $\lim _{\alpha, \gamma \rightarrow 1} F(\alpha, \gamma) \approx$ 4. Together with the additional prefactors $\pi /[6 \sqrt{2} \zeta(3 / 2)] \approx$ 0.14 in Eq. (52) this yields $\chi_{T} \lesssim 0.56 \chi_{0}$.

In Fig. 7 the flux dependence of the bulk result, Eq. (52), is compared with the numerically calculated contribution from the conduction and valence band to $\chi$ of (a) an armchair and (b) a zigzag triangular quantum dot at $\mu=0$. The thermal energies of the bulk systems are chosen such that $\beta^{\text {(bulk) }}\left\langle\Delta \bar{E}^{\text {(bulk) }}\right\rangle_{\phi} \approx \beta^{(a c, z z)}\left\langle\Delta \bar{E}^{(a c, z z)}\right\rangle_{\phi}$. By choosing lower thermal energies finite-size effects gain importance and deviations from the bulk theory emerge as can be seen from Fig. 8: The susceptibilities $\chi_{T}$ of the quantum dots exhibit oscillatory behavior which becomes all the more pronounced, as the thermal energies tend to lower values. In this case all parameters are chosen as in Fig. 7 but the thermal energy of the quantum dots is one order of magnitude smaller, i.e., $1 / \beta^{(a c, z z)} \approx 10^{-3} t$. For these parameters, the function $F(\alpha, \gamma)$, Eq. (53), reaches positive values only in the considered flux range. Therefore $\chi_{T}$, Eq. (52), is diamagnetic. This holds also true for the numerically calculated contribution $\chi_{T}$ of the triangular quantum dots. From the definition (35) of $\alpha \propto \Delta_{L L} / \mu$ one expects the bulk effects to dominate over finite-size signatures and therefore good agreement of the numerical data with the bulk calculations for $\phi \gtrsim 15 \phi_{0}$. This is confirmed by Figs. 7 and 8 .

For lower values of $\phi$ Eq. (51) is no longer valid yielding deviations from the tight-binding calculations as the oscillatory modulations of $\chi_{T}$ demonstrate in Fig. 8. These oscillations are smeared out due to the larger thermal energies chosen in Fig. 7. In both figures $\chi_{T}$ of the quantum dots reaches zero for $\varphi \approx 0$ and $\chi_{T}$ is moreover suppressed on a finite flux interval, $\phi \lesssim 3 \phi_{0}$ in Fig. 8(a) and $\phi \lesssim 7 \phi_{0}$ in Fig. 8(b), respectively. This behavior can be understood in view of the energy spectra of the quantum dots, Fig. 2. In each case there is a small gap between $E=0$ and the first nonzero energy level as a signature 


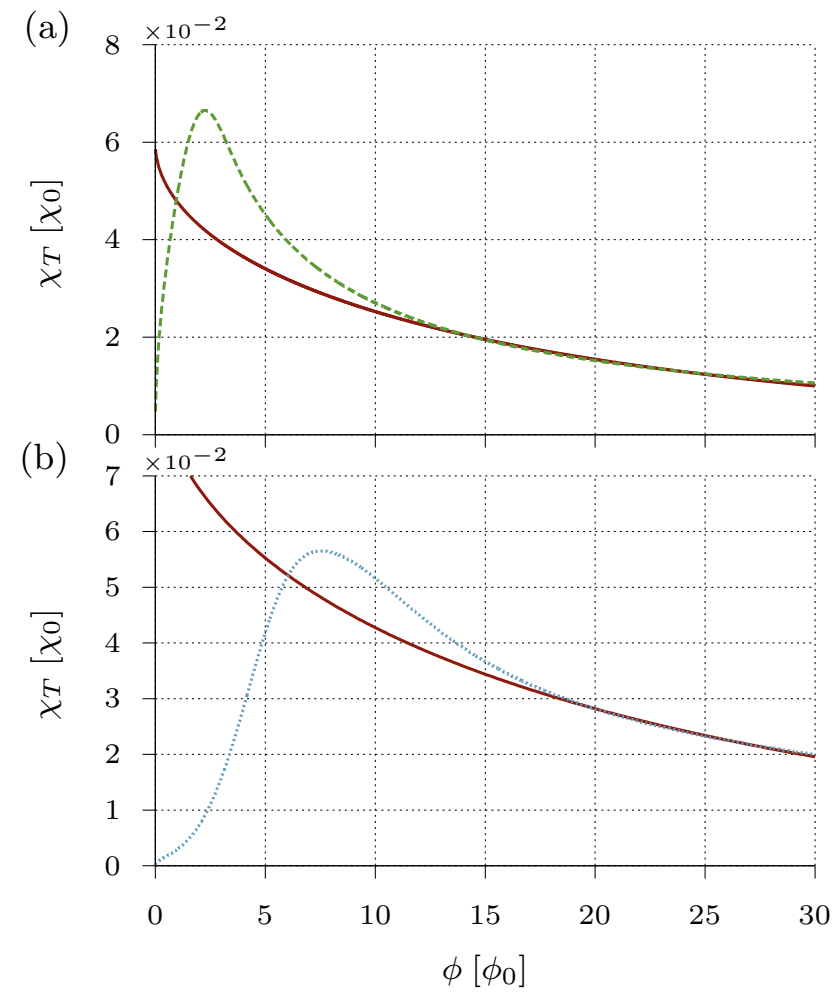

bulk

FIG. 7. (Color online) Flux dependence of the temperaturedependent susceptibility contribution $\chi_{T}$ of bulk graphene compared with the numerically calculated contribution of triangular nanostructures with (a) armchair and zigzag (b) edges of side length $\mathcal{L} \approx 100 a$. The chemical potential is chosen at $\mu=0$ and the thermal energies are $1 / \beta^{(a c, z z)} \approx 10^{-2} t$ and $1 / \beta^{\text {(bulk) }} \approx 1.5 \times 10^{-1} t$ in panel (a) and $1 / \beta^{\text {(bulk) }} \approx 1.7 \times 10^{-1} t$ in panel (b), such that Eq. (18) holds true. The scaling factors $\gamma^{(a c)} \approx 2.5 \times 10^{-2}$ and $\gamma^{(z z)} \approx 3.8 \times 10^{-2}$ are obtained by fitting.

of confinement. For thermal energies smaller than this gap and $\mu=0$ there are no occupied states above the Dirac point besides the edge states of the zigzag quantum dot contributing $\varphi$ linear to $\Omega_{T}$ and yielding $\chi_{T}=0$. In the case of the armchair quantum dot $\Omega_{T}$ and therefore $\chi_{T}$ vanish completely in this specific parameter range.

\section{Regime: $\gamma<1$ and arbitrary $\alpha$}

If the thermal energy of the system is larger than the level spacing, also states above the Fermi level are occupied implying that tuning the chemical potential or the magnetic field does not lead to a discontinuity of the corresponding contribution to the grand potential. As a consequence the susceptibility is expected to be a smooth function of these parameters. In this parameter range the magnetic flux and the thermal energy can be chosen in such a way that the Landau level clustering in the quantum dot spectra is pronounced enough to make the bulk theory valid, on the one hand, and effectively wash out the finite-size signatures on the other hand. Hence one can expect good agreement of the bulk theory with the susceptibility of the quantum dots.

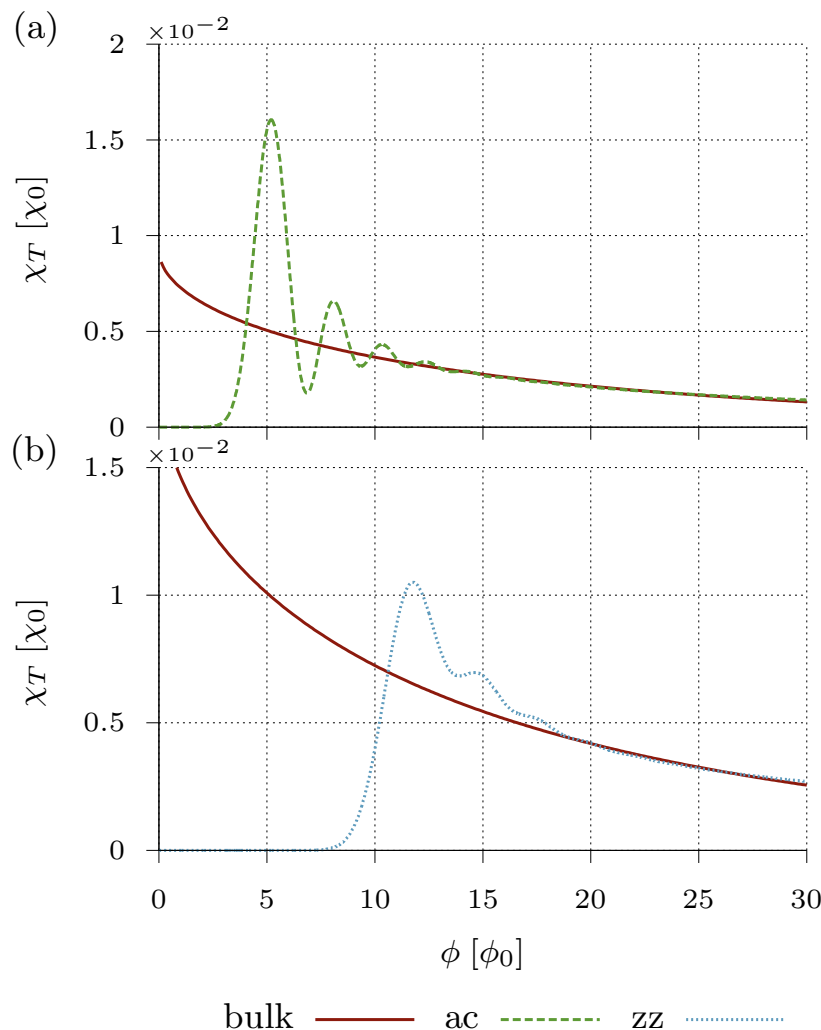

FIG. 8. (Color online) Same as Fig. 7 for smaller thermal energies $1 / \beta^{(a c, z z)} \approx 10^{-3} t$ and $1 / \beta^{\text {(bulk })} \approx 1.44 \times 10^{-1} t$ in panel (a) and $1 / \beta^{\text {(bulk) }} \approx 1.42 \times 10^{-1} t$ in panel (b), such that Eq. (18) holds true. The scaling factors $\gamma^{(a c)} \approx 3.9 \times 10^{-3}$ and $\gamma^{(z z)} \approx 7.8 \times 10^{-3}$ are obtained by fitting.

Using again the decomposition of the Fresnel integral into the smooth and oscillatory part, one can start from representation Eq. (38) of the field-dependent part of $\Omega$. Substituting $E=2 / \beta x+\mu$ gives

$$
\begin{aligned}
\tilde{\Omega}= & \frac{K}{2 \sqrt{2} \pi} \varphi^{3 / 2} \sum_{m=1}^{\infty} \frac{1}{\sqrt{m}^{3}} \operatorname{Im}\left[\int_{0}^{\infty} d u \frac{1}{\sqrt{u}(u-i)}\right. \\
& \left.\times \int_{-\infty}^{\infty} d x \operatorname{sech}^{2}(x) e^{-u \pi m[2(x / \gamma)+1 / \alpha]^{2}} e^{i \pi m[2(x / \gamma)+1 / \alpha]^{2}}\right] .
\end{aligned}
$$

For $\gamma<1$ the complex phase rapidly oscillates as a function of $x$ for all values of $\alpha$. Therefore the second integral can be solved within stationary phase approximation:

$$
\int_{-\infty}^{\infty} d x \operatorname{sech}^{2}(x) e^{i \pi m[2(x / \gamma)+1 / \alpha]^{2}} \approx \frac{|\gamma|}{2 \sqrt{m}} \operatorname{sech}^{2}\left(\frac{\gamma}{2 \alpha}\right) e^{i(\pi / 4)} .
$$

Using $\int_{0}^{\infty} d u[\sqrt{u}(u-i)]^{-1}=\pi \exp (-i \pi / 4)$ in (54) yields

$$
\begin{aligned}
\tilde{\Omega} & \approx \frac{K}{4 \sqrt{2}} \sqrt{\varphi}^{3}|\gamma| \operatorname{sech}^{2}\left(\frac{\gamma}{2 \alpha}\right) \sum_{m=1}^{\infty} \frac{1}{m^{2}} \\
& =\frac{g}{\mathcal{A}}\left(\hbar v_{F}\right)^{2} \frac{\pi^{2}}{12} \beta \operatorname{sech}^{2}\left(\frac{\gamma}{2 \alpha}\right) \varphi^{2},
\end{aligned}
$$


where $\sum_{m=1}^{\infty} m^{-2}=\pi^{2} / 6$ is used. The corresponding expression for the total orbital susceptibility reads

$$
\begin{aligned}
\chi(\mu) & =-\frac{\mu_{0} g}{\phi_{0}^{2}}\left(\hbar v_{F}\right)^{2} \frac{\pi^{2}}{6} \beta \operatorname{sech}^{2}\left(\frac{\mu \beta}{2}\right) \\
& =\chi_{0}(B) \times \frac{\sqrt{2} \pi^{2}}{9 \zeta\left(\frac{3}{2}\right)} \gamma \operatorname{sech}^{2}\left(\frac{\gamma}{2 \alpha}\right) .
\end{aligned}
$$

In this regime the divergent contribution $\chi_{0}$ of the filled valence band is compensated by the contribution $\chi_{T}$ of the thermally excited charge carriers leading to a distinctly diamagnetic and moreover flux independent magnetic response. This result can also be found in the literature [2-5]. The contribution $\chi_{T}=\chi-\chi_{0}$ can be extracted from Eq. (59) reading

$$
\chi_{T}(\mu)=-\chi_{0}(B)\left[1-\frac{\sqrt{2} \pi^{2}}{9 \zeta\left(\frac{3}{2}\right)} \gamma \operatorname{sech}^{2}\left(\frac{\gamma}{2 \alpha}\right)\right] .
$$

Since $\sqrt{2} \pi^{2} /[9 \zeta(3 / 2)] \approx 0.6$ and $\operatorname{sech}^{2}(x) \leqslant 1, \forall x \in \mathbb{R}$ the contribution $\chi_{T}$ exhibits paramagnetic behavior in this parameter range. The comparison of this bulk contribution with numerical data for the triangular armchair and zigzag quantum dot in Figs. 9(a) and 9(b) shows perfect agreement as expected at larger fluxes.

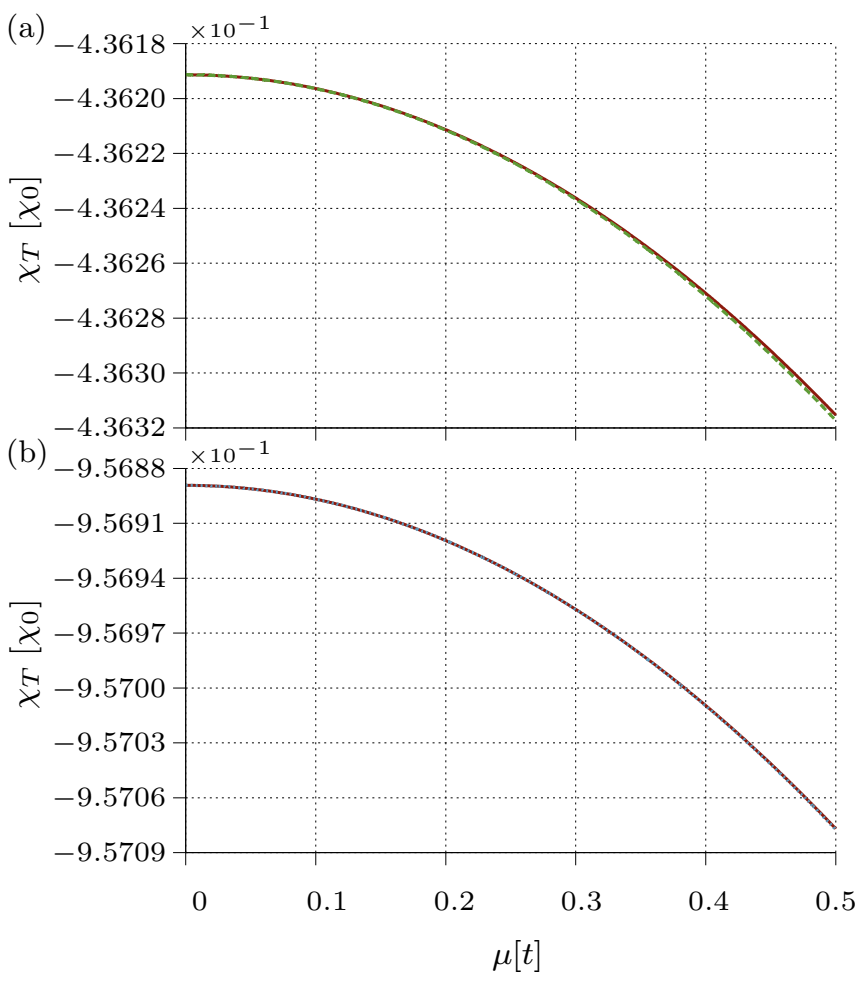

bulk $\longrightarrow$ ac $------\cdot$ zz

FIG. 9. (Color online) Comparison of the temperaturedependent susceptibility contribution $\chi_{T}$ for bulk graphene with that of a triangular armchair (a) and zigzag (b) graphene flake as a function of the chemical potential at a magnetic flux of $\phi=5 \phi_{0}$ and $1 / \beta^{(a c, z z)}=5 t$. The corresponding values used in the analytic expression for the bulk are $1 / \beta^{\text {(bulk) }} \approx 2.1 t$ in (a) and $1 / \beta^{\text {(bulk) }} \approx 2.9 t$ in (b). The fitted scaling factors are $\gamma^{(a c)}=0.44, \gamma^{(z z)}=1.7$.
To fulfill $\gamma<1$, i.e., $\sqrt{\mathcal{A} /(2 \pi) \varphi}<k_{B} T /\left(\hbar v_{F}\right)$, in the limit of very low temperatures requires that $\left|E_{n}\right|$, Eq. (12), tend to zero even for large Landau indices $n$. Hence a change in the magnetization of bulk graphene due to weakly thermally excited charge carriers can only occur for Fermi energies close to the Dirac point. For $T \rightarrow 0$ this leads to a sharply peaked susceptibility at $\mu=0$. In view of Eq. (32), this can be deduced from Eq. (58) yielding the well-known expression [2,4-11]

$$
\chi(\mu) \stackrel{\beta \rightarrow \infty}{\longrightarrow}-\frac{\mu_{0} g}{\phi_{0}^{2}}\left(\hbar v_{F}\right)^{2} \frac{2 \pi^{2}}{3} \delta(\mu) .
$$

This limit is not truly reachable numerically for the finite systems considered since the Landau-level structure is not pronounced enough as can be seen from Fig. 2.

Another limit of physical relevance concerns $\mu \rightarrow 0$ or $\alpha \rightarrow \infty$. In this limit the total orbital susceptibility reads

$$
\chi(\mu) \stackrel{\mu \rightarrow 0}{\longrightarrow}-\frac{\mu_{0} g}{\phi_{0}^{2}}\left(\hbar v_{F}\right)^{2} \frac{\pi^{2}}{6} \beta \propto-\frac{1}{k_{B} T} .
$$

This typical temperature dependence, already known in the literature [3], is also affirmed by the numerical data; see Fig. 10. The double logarithmic graphs in the insets show clearly the

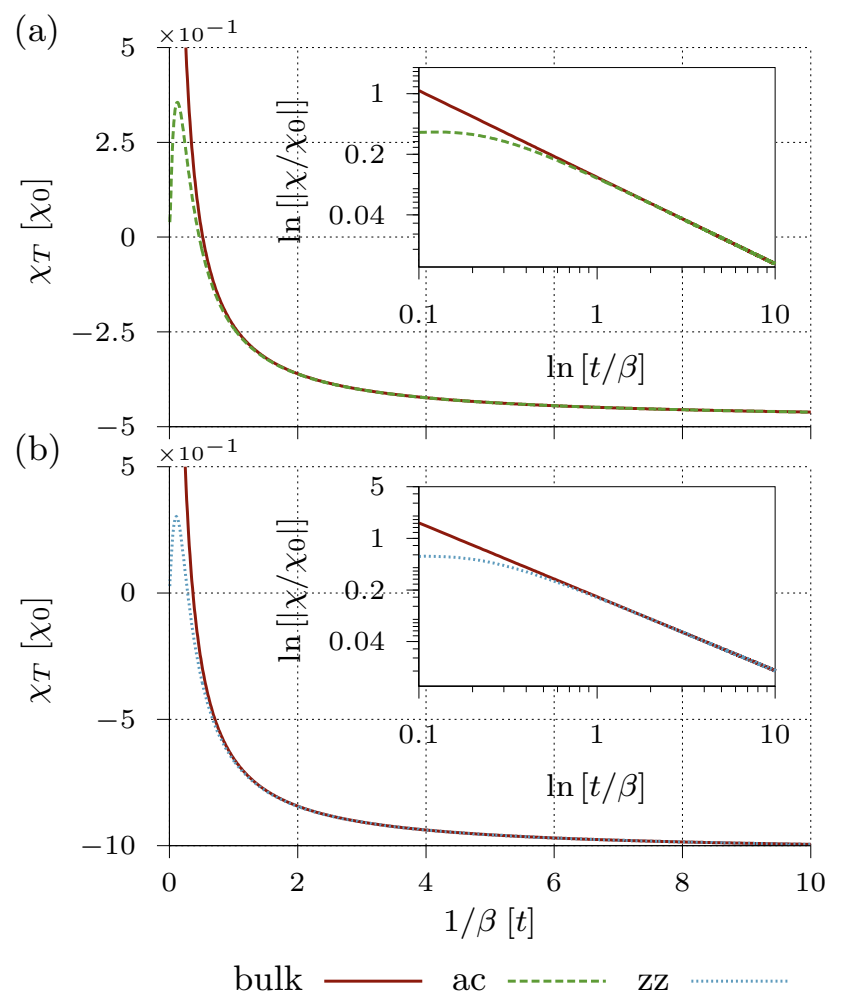

FIG. 10. (Color online) Comparison of the numerical data of the orbital magnetic susceptibility contribution $\chi_{T}$ of a triangular armchair (a) and zigzag (b) graphene flake at $\phi=5 \phi_{0}$ with the analytic result for bulk graphene in the limit $\mu \rightarrow 0$. In both cases the correspondence is convincing for $t / \beta>1$ as for lower thermal energies this approximation loses validity. The scaling factors attain $\gamma^{(a c)} \approx 5.8$ and $\gamma^{(z z)} \approx 8.6$ which is in agreement with the condition $\left|\bar{E}^{\text {(bulk) }}-\bar{E}^{(a c)}\right|<\left|\bar{E}^{(\text {bulk })}-\bar{E}^{(z z)}\right|$. The insets show both the full orbital magnetic susceptibility $\chi$ in a double logarithmic plot and confirm the scaling behavior $\chi \propto-\beta$ at the Dirac point. 
$1 / k_{B} T$ dependence in the limit $\mu \rightarrow 0$ (for $\phi=5 \phi_{0}$ ). The difference between the bulk theory and the numerical data for small thermal energies reflects, on the one hand, the limit of validity of the analytical approximation for $\gamma<1$; on the other hand, it is a signature of finite-size effects which gain importance in the low-temperature limit.

\section{OSCILLATORY FINITE-SIZE EFFECTS FOR GRAPHENE NANOSTRUCTURES}

\section{A. General semiclassical framework}

Semiclassical periodic orbit theories offer a distinguished way to analytically describe finite-size effects encoded in the energy spectra of spatially confined systems of arbitrary shape. Boundary effects are incorporated in the semiclassical approximation of the oscillatory part of the DOS, $\rho_{\mathrm{sc}}^{\text {osc }}(E)$. One important criteria for applying such semiclassical approximations, the Gutzwiller trace formula [49] for chaotic classical dynamics or the Berry-Tabor trace formula [50] for regular classical dynamics, requires that the linear system size lies in a mesoscopic regime, $k \mathcal{L} \gg 1$, where $k=E /\left(\hbar v_{F}\right)$ is the Fermi wave number. In general, $d_{\mathrm{sc}}^{\text {osc }}(E)$ is of the form

$$
\begin{gathered}
d_{\mathrm{sc}}^{\mathrm{osc}}(E)=\sum_{\gamma} d_{\mathrm{sc}, \gamma}^{\mathrm{osc}}(E), \\
d_{\mathrm{sc}, \gamma}^{\mathrm{osc}}(E) \propto \operatorname{Re} D_{\gamma} e^{(i / \hbar) S_{\gamma},}
\end{gathered}
$$

where the sum runs over infinitely many classical periodic orbits $\gamma$ with classical action $S_{\gamma}=\oint_{\gamma} d \mathbf{q} \cdot \mathbf{p}=p \mathcal{L}_{\gamma}$ and length $\mathcal{L}_{\gamma}$. The exact form of the classical amplitude $D_{\gamma}$ sensitively depends on the specific geometry of the system and can be calculated either within the recipe given by Gutzwiller [49] in the case of nonintegrable classical dynamics or within the recipe of Berry and Tabor [50] when the classical dynamics is integrable. In the latter case, relevant in the following, the summation over $\gamma$ in Eq. (63) runs over families of degenerate orbits, as depicted in Fig. 11(a) for a disk geometry. This degeneracy of orbits in a regular billiard can be described in terms of continuous symmetry groups $G$ such that the members of a specific orbit family are related to each other through the action of a group element $g$ of $\mathbb{G}$. This is already included in the Berry-Tabor trace formula $[50,51]$ for field-free regular

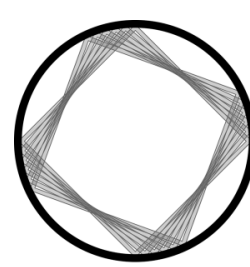

(a)

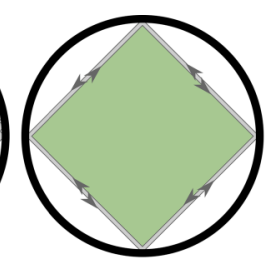

(b) $B \approx 0$

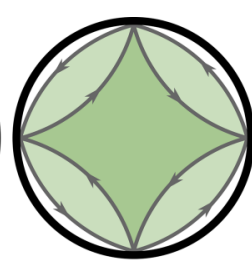

(c) $B>0$
FIG. 11. (Color online) Classical periodic orbits in the circular billiard. Panel (a) shows representatives of one fundamental orbit family. Panels (b) and (c): Pairs of counterpropagating orbits in the presence of a perpendicular magnetic field. For weak magnetic fields, panel (b), the bending of the classical orbits can be neglected and the enclosed areas (green shaded) are approximately equal. Panel (c) shows the same orbit pair for stronger magnetic field. systems. In the case of small symmetry breaking [52-56], as it is caused by a weak external magnetic field, one has to take these degeneracies separately into account as discussed in Sec. IV B. Therefore, we will associate an orbit family $\gamma$ with the corresponding element $g$ of the underlying symmetry group $\mathbb{G}$ if necessary, i.e., $\gamma \mapsto \gamma(g)$.

In Refs. [20,21,57] the authors show in a general way how the trace formulas for "Schrödinger billiards" with classically regular or chaotic dynamics can be extended to an arbitrary shaped, field-free graphene flake including the most common types of boundaries, i.e., zigzag, armchair, and infinite-masstype edges. Resembling Eq. (63) the semiclassical trace formulas for graphene read

$$
\rho_{\mathrm{sc}}^{\mathrm{osc}}(E)=\sum_{\gamma} \rho_{\mathrm{sc}, \gamma}^{\mathrm{osc}}(E), \quad \rho_{\mathrm{sc}, \gamma}^{\mathrm{osc}}(E) \propto d_{\mathrm{sc}, \gamma}^{\mathrm{osc}}(E) \operatorname{Tr} K_{\gamma},
$$

where $d_{\mathrm{sc}, \gamma}^{\text {osc }}$ is given by Eq. (63) of the corresponding Schrödinger system. Hence, the $d_{\mathrm{sc}, \gamma}^{\text {osc }}$ contain all information about the orbital dynamics in the graphene system. The additional factor $\operatorname{Tr} K_{\gamma}$ denotes a trace over the pseudospin propagator $K_{\gamma}$ of the orbit $\gamma$ and contains only graphene specific information about the boundary. In Refs. [21,57] a general expression for $\operatorname{Tr} K_{\gamma}$ of an orbit, with $N_{\gamma}$ reflections at the boundaries, is derived, yielding

$$
\operatorname{Tr} K_{\gamma}=4 f_{\gamma} \cos \left(\theta_{\gamma}+\frac{\pi}{2} N_{\gamma}\right) \cos \left(2 K \Lambda_{\gamma}+\vartheta_{\gamma}+\frac{\pi}{2} N_{\gamma}\right),
$$

if the total number of reflections on armchair edges, $N_{a c}$, is even and $\operatorname{Tr} K_{\gamma}=0$ otherwise. The prefactor is defined as $f_{\gamma}=i^{3 N_{\gamma}-N_{z z}}$, where $N_{z z}$ denotes the number of reflections on zigzag edges. $\theta_{\gamma}=\sum_{i=1}^{N_{\gamma}} \theta_{i}$ is the sum over all reflection angles along the orbit $\gamma . K=4 \pi /(3 a)$ denotes the distances between the Dirac points and the $\Gamma$ point of the Brillouin zone. $\Lambda_{\gamma}=$ $\sum_{i=1}^{N_{a c} / 2}\left(x_{2 i-1}-x_{2 i}\right)$ is the sum over the distance between two subsequent reflections on armchair edges. Further $\vartheta_{\gamma}=$ $\sum_{i=1}^{N_{z z}}(-1)^{s_{i}} \vartheta_{i}$ denotes the sum over $z z$ reflection angles $\vartheta_{i}$, where $\vartheta_{i}= \pm \theta_{i}$ for reflection on $A$ and $B$ edges, respectively, and $s_{i}$ is the number of $a c$ reflections occurring after the $z z$ reflection $i$. One finds [21,57] $\operatorname{Tr} K_{\gamma}=\operatorname{Tr} K_{\gamma^{-1}}$ where $\gamma^{-1}$ denotes the time-reversed partner of orbit $\gamma$.

\section{B. Semiclassical approximation of the orbital magnetic susceptibility}

In Ref. [16] the authors showed how the semiclassical theory of integrable and nonintegrable billiard systems with parabolic dispersion can be extended to include the effect of a homogeneous, constant magnetic field. Due to the formal similarity of the trace formulas of systems with parabolic dispersion, Eq. (63), and graphene, Eq. (65), the techniques used in Ref. [16] can be readily transferred. We will focus on the low-field regime where the classical cyclotron radius $R_{c}=k l_{B}^{2}$ is much larger than the linear system size, i.e., $R_{c} \gg \mathcal{L}$. In the following we will consider quantum dots with corresponding regular classical dynamics in the field-free case. A derivation of orbital magnetic properties of cavities with chaotic underlying dynamics can be derived correspondingly. Following Refs. [52,53], we treat the weak magnetic field 
perturbatively, such that the classical Hamiltonian of the system,

$$
\mathcal{H}=\frac{[\mathbf{p}-e \mathbf{A}(\mathbf{q})]^{2}}{2 m}+V(\mathbf{q}),
$$

can be decomposed into the unperturbed part, $\mathcal{H}_{0}=$ $\mathbf{p}^{2} /(2 m)+V(\mathbf{q})$, and the small perturbation $-\frac{1}{m} \mathbf{p} \cdot \mathbf{A}(\mathbf{q})$. To leading perturbative order the action difference between an orbit in the perturbed and the unperturbed system reads [52,53]

$$
\delta S_{\gamma} \approx e \int_{\gamma} d \mathbf{q} \cdot \mathbf{A}(\mathbf{q})=e \mathbf{B} \cdot \mathcal{A}_{\gamma},
$$

with $\mathcal{A}_{\gamma}$ the directed, enclosed area of the unperturbed orbit $\gamma$. In Refs. [16,36,52] it is moreover shown that in the presence of a weak magnetic field the trace formula (63) for the field-free Schrödinger system is modified to

$$
\begin{aligned}
d_{\mathrm{sc}}^{\mathrm{osc}}(E, B) & =\sum_{\gamma} d_{\mathrm{sc}, \gamma}^{\mathrm{osc}}(E, B), \\
d_{\mathrm{sc}, \gamma}^{\mathrm{osc}}(E, B) & \propto \operatorname{Re}\left[D_{\gamma} e^{(i / \hbar) S_{0, \gamma}} \times \mathcal{M}_{\gamma}(B)\right],
\end{aligned}
$$

with the field-dependent modulation factor

$$
\begin{aligned}
\mathcal{M}_{\gamma}(B) & =\frac{1}{V_{g}} \int_{\mathbb{G}} d \mu(g) e^{(i / \hbar) \delta S_{\gamma(g)}} \\
& =\frac{1}{V_{g}} \int_{\mathbb{G}} d \mu(g) e^{i\left(2 \pi / \phi_{0}\right) \mathbf{B} \cdot \mathcal{A}_{\gamma(g)}} .
\end{aligned}
$$

The index $g$ represents an element of the symmetry group $\mathbb{G}$ characterizing the degeneracy of orbits $\gamma(g)$ in one specific orbit family. Since $\mu(g)$ is the Haar measure [58] of $\mathbb{G}$, the normalization factor $V_{g}=\int_{\mathbb{G}} d \mu(g)$ can be understood as the volume of $\mathbb{G}$. Since $d_{\mathrm{sc}}^{\text {osc }}$ contains all information of the orbital dynamics, including the influence of the $B$ field, we can adapt Eq. (69) and derive the oscillatory part of the DOS for a regular graphene cavity in a weak magnetic field in semiclassical approximation:

$$
\begin{gathered}
\rho_{\mathrm{sc}}^{\mathrm{osc}}(E, B)=\sum_{\gamma} \rho_{\mathrm{sc}, \gamma}^{\mathrm{osc}}(E, B), \\
\rho_{\mathrm{sc}, \gamma}^{\mathrm{osc}}(E, B) \propto d_{\mathrm{sc}, \gamma}^{\mathrm{osc}}(E, B) \operatorname{Tr} K_{\gamma} .
\end{gathered}
$$

Equation (71) is applicable to both systems that remain integrable in a weak magnetic field and systems which are no longer integrable due to the symmetry breaking caused by a weak magnetic field, e.g., a rectangular quantum dot considered in Sec. IV D. The lengths of time-reversed partner orbits or families, $\gamma$ and $\gamma^{-1}$ (for $B=0$ ), are equal, but the directed, enclosed areas have opposite signs due to the propagation direction, i.e., $\mathcal{L}_{\gamma}=\mathcal{L}_{\gamma^{\prime}}$ and $\mathcal{A}_{\gamma}=-\mathcal{A}_{\gamma^{\prime}}$. The contribution of these orbit pairs to the DOS can be combined to

$$
\rho_{\mathrm{sc}, \gamma}^{\mathrm{osc}}(E, B)+\rho_{\mathrm{sc}, \gamma^{\prime}}^{\mathrm{osc}}(E, B)=2 \rho_{\mathrm{sc}, \gamma}^{\mathrm{osc}}(E) \times \mathcal{C}_{\gamma}(B),
$$

where $\rho_{\mathrm{sc}, \gamma}^{\mathrm{osc}}(E)$ is the contribution (65) of the orbit family $\gamma$ to $\rho_{\mathrm{sc}}^{\mathrm{osc}}$ in the field-free system and

$$
\mathcal{C}_{\gamma}(B)=\frac{1}{V_{g}} \int_{\mathbb{G}} d \mu(g) \cos \left(\frac{2 \pi}{\phi_{0}} \mathbf{B} \cdot \mathcal{A}_{\gamma(g)}\right) .
$$

The field dependence of the DOS and therefore of related observables such as the magnetic susceptibility is governed by dephasing between time-reversed orbit families and affected by dephasing between different members of a given orbit family induced by the magnetic field. From definition (29) of the grand potential one can deduce the semiclassical approximation of the oscillatory part [16]

$$
\Omega_{\mathrm{sc}}^{\mathrm{osc}}(\mu, B)=\int_{-\infty}^{\infty} d E \mathcal{N}_{\mathrm{sc}}^{\mathrm{osc}}(E, B) f^{\prime}(E-\mu),
$$

where $\mathcal{N}_{\mathrm{sc}}^{\mathrm{osc}}$ is obtained from $\rho_{\mathrm{sc}}^{\text {osc }}$ after integrating twice by parts. For the contribution of the orbit family $\gamma$ to the oscillatory DOS, $\rho_{\mathrm{sc}, \gamma}^{\mathrm{osc}}$, one finds [16]

$$
\mathcal{N}_{\mathrm{sc}, \gamma}^{\mathrm{osc}}(E, B)=-\left(\frac{\hbar}{d S_{\gamma} / d E}\right)^{2} \rho_{\mathrm{sc}, \gamma}^{\mathrm{osc}}(E, B) .
$$

The energy integral (74) is of the form of Eq. (39) and solved as described in Appendix A of Ref. [16]. Using Eq. (40) and $d S_{\gamma} / d E=\tau_{\gamma}=\mathcal{L}_{\gamma} / v_{F}$ one eventually finds

$$
\Omega_{\mathrm{sc}}^{\mathrm{osc}}(\mu, B) \approx \sum_{\gamma}\left(\frac{\hbar v_{F}}{\mathcal{L}_{\gamma}}\right)^{2} \rho_{\mathrm{sc}, \gamma}^{\mathrm{osc}}(\mu, B) R_{T}\left(\frac{\mathcal{L} \gamma}{\mathcal{L}_{c}}\right) .
$$

At finite $T$ the sum converges due to the exponential suppression of orbit families with $\mathcal{L}_{\gamma}>\mathcal{L}_{c}=\hbar v_{F} \beta / \pi$ encoded in $\mathrm{R}_{T}$, Eq. (41). Taking twice the $B$-field derivative one finds the semiclassical, oscillatory contribution to the orbital susceptibility of a graphene nanostructure with underlying regular classical dynamics:

$$
\begin{aligned}
\chi_{\mathrm{sc}}^{\mathrm{osc}}(\mu, B)= & -\frac{\mu_{0}}{\mathcal{A}} \sum_{\gamma}\left(\frac{\hbar v_{F}}{\mathcal{L}_{\gamma}}\right)^{2} R_{T}\left(\frac{\mathcal{L}_{\gamma}}{\mathcal{L}_{\rfloor}}\right) \\
& \times f_{\gamma} \rho_{\mathrm{sc}, \gamma}^{\mathrm{osc}}(\mu) \frac{\partial^{2}}{\partial B^{2}} \mathcal{C}_{\gamma}(B) .
\end{aligned}
$$

Here, the sum involves one propagation direction of orbit families $\gamma$. Time-reversed partners are considered by the factor $f_{\gamma}=2$. The magnetic phase factor $\mathcal{C}_{\gamma}$, Eq. (73), implies that only orbits contribute to $\chi_{\mathrm{sc}}^{\text {osc }}$ that enclose a finite area in the field-free case, and hence self-retracing orbits $\left(f_{\gamma}=1\right)$ do not contribute. We note that the same formal expression (77) holds true for Schrödinger-type systems and graphene, since the graphene-specific relevant information is implicitly contained in $\rho_{\mathrm{sc}, \gamma}^{\mathrm{osc}}$.

In the following we compare these predictions for the orbital magnetic response with quantum-mechanical results within the effective Dirac model (Sec. IV C) and full tight-binding calculations (Sec. IV D).

\section{Circular billiard with infinite-mass-type edges}

The first representative system we analyze is a disk-shaped graphene quantum dot with infinite-mass-type edges. Due to its rotational symmetry there is a separable quantum-mechanical solution within the Dirac approximation even in the presence of a magnetic field. The resulting quantization condition reads [59,60]

$$
J_{\bar{m}}\left(k_{\bar{m} n} R\right)=\tau J_{\bar{m}+1}\left(k_{\bar{m} n} R\right) .
$$




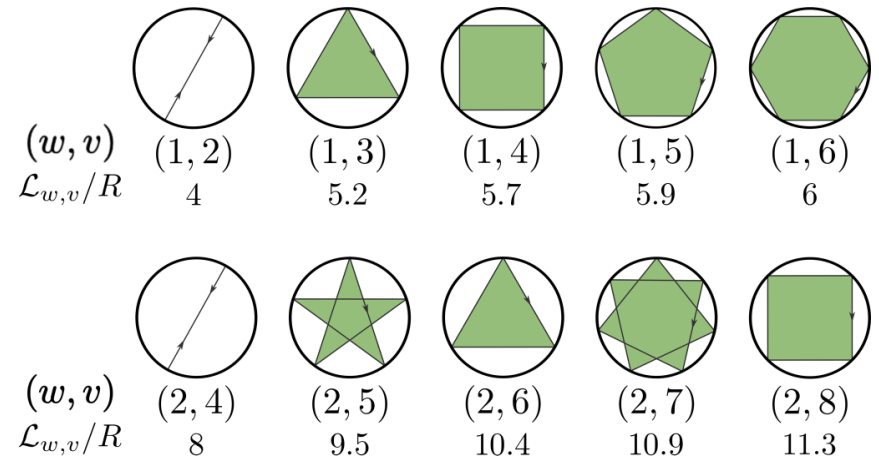

FIG. 12. (Color online) Trajectories representing families of classical periodic orbits in the disk billiard. $w$ denotes the winding number, whereas $v$ labels the total number of boundary reflections. Enclosed areas are marked in green.

Here, $\tau= \pm 1$ labels the two valleys of the graphene Brillouin zone, $R$ is the disk radius, and $J_{v}(x)$ denotes the $v$ th-order Bessel function of the first kind [48]. The index $\bar{m}=m+\phi / \phi_{0}$ includes the magnetic flux $\phi$ and the azimuthal orbital angular momentum quantum number $m=0, \pm 1, \ldots$. The second quantum number $n \in \mathbb{Z}$ counts (for a given $\bar{m}$ ) the solutions $k_{\bar{m} n}$ to Eq. (78) which are obtained numerically. Each energy level has a twofold spin degeneracy. Based on Eq. (78), one can calculate the orbital magnetic susceptibility quantum mechanically according to Eq. (6).

The semiclassical properties of the disk cavity with infinitemass-type edges have already been considered (for $B=0$ ) in Refs. [20,57]. In order to compute its magnetic properties within semiclassical approximation, we combine these results with results adapted from Ref. [16], where $\chi_{\mathrm{sc}}^{\text {osc }}$ for the Schrödinger disk billiard was derived. For the disk geometry, one can characterize the periodic-orbit families by their winding number $w$ and their total number $v$ of reflections at the boundary (with $v \geqslant 2 w$ ). The sign of $w$ defines the direction of rotation. A few representative periodic-orbit families are depicted in Fig. 12 for $w=1,2$, together with their lengths $\mathcal{L}_{w, v}$ and the enclosed areas $\mathcal{A}_{w, v}$ (green shaded). They can be calculated within basic geometry yielding [16]

$$
\begin{aligned}
& \mathcal{L}_{w, v}=2 v R \sin \left(\left|\pi \frac{w}{v}\right|\right), \\
& \mathcal{A}_{w, v}=\mathcal{A} \frac{v}{2 \pi} \sin \left(2 \pi \frac{w}{v}\right),
\end{aligned}
$$

with area $\mathcal{A}=\pi R^{2}$. The trace over the pseudospin propagator for an orbit family characterized by the tupel $(w, v)$ can be calculated from Eq. (66) and reads [20,57]

$$
\operatorname{Tr} K_{w, v}=g \cos \left(v \theta_{w, v}\right) \begin{cases}(-1)^{v / 2} & \text { for even } v, \\ 0 & \text { for odd } v\end{cases}
$$

with the reflection angle $\theta_{w, v}=[\operatorname{sgn}(w) / 2-w / v] \pi$. Due to pseudospin interference only orbits with an odd number of reflections contribute to the DOS, in contrast to the corresponding Schrödinger system $[16,36]$. Therefore, the entire field-dependent, oscillatory contribution to the DOS reads

$$
\begin{aligned}
\rho_{\mathrm{sc}}^{\mathrm{osc}}(E, B)= & \frac{2}{\hbar v_{F}} \sqrt{\frac{k}{2 \pi}} \sum_{w=1}^{\infty} \sum_{\substack{v \geqslant 2 w \\
\text { even }}}^{\infty}(-1)^{w+v / 2} \frac{f_{w, v}}{v^{2}} \mathcal{L}_{w, v}^{3 / 2} \\
& \times \sin \left(k \mathcal{L}_{w, v}+\frac{3}{4} \pi\right) \mathcal{C}_{w, v}(B) .
\end{aligned}
$$

Owing to the rotational symmetry, the $B$-field induced modulation of each contribution is only due to dephasing between time-reversed orbits such that the magnetic phase factor reads [16]

$$
\mathcal{C}_{w, v}(B)=\frac{1}{2 \pi} \int_{0}^{2 \pi} d \varphi \cos \left(\frac{\mathcal{A}_{w, v}}{l_{B}^{2}}\right)=\cos \left(\frac{\mathcal{A}_{w, v}}{l_{B}^{2}}\right) .
$$

Together with Eq. (77), one then finds for the semiclassical approximation of the oscillatory contribution to the orbital magnetic susceptibility [in terms of $\chi_{0}$, Eq. (25)]:

$$
\begin{aligned}
\chi_{\mathrm{sc}}^{\mathrm{osc}}(\mu, B)= & -\chi_{0}(B) \times \frac{8 \pi^{3 / 2}}{3 \zeta(3 / 2)} \frac{R}{l_{B}} \sqrt{k_{F} R} \\
& \times \sum_{w=1}^{\infty} \sum_{\substack{v \geqslant 2 w \\
\text { even }}}^{\infty} \frac{(-1)^{w+v / 2}}{v^{2}}\left(\frac{\mathcal{A}_{w, v}}{R^{2}}\right)^{2} \sqrt{\frac{R}{\mathcal{L}_{w, v}}} \\
& \times \sin \left(k_{F} \mathcal{L}_{w, v}+\frac{3}{4} \pi\right) \mathcal{C}_{w, v}(B) R_{T}\left(\frac{L_{w, v}}{\hbar v_{F}}\right) .
\end{aligned}
$$

Since bouncing-ball orbits $(w, 2 w)$ do not enclose a finite area in the weak-field limit they are not considered in $\chi_{\mathrm{sc}}^{\text {osc }}$, and we absorbed the factor $f_{w, v}=2$ into the overall prefactor. Expression (84) demonstrates that the confinement-induced magnetic response of an integrable geometry is parametrically larger (by a factor $\sqrt{k_{\mathrm{F}} R}$ ) than the bulk value $\chi_{0}$.

Figure 13(a) shows the length spectrum resulting from the Fourier transform of the quantum-mechanical result for $\chi^{\text {osc }}(\mu)$ at $B=0$. One can clearly identify the peak positions with the lengths $\mathcal{L}_{w, v}$ of the shortest contributing orbits as expected from the semiclassical formula (84). Green arrows mark the lengths of those orbits that do not contribute due to destructive pseudospin interference according to Eq. (81). Apparently, as visible in Fig. 13(a), also bouncing-ball orbits $(w, 2 w)$ yield a contribution to the quantum-mechanical result $\chi^{\text {osc }}$, even though, according to Eq. (83), their semiclassical contribution vanishes at weak fields if bending of the trajectories is not included. The temperature used in Fig. 13 is equivalent to a short cutoff length of $\mathcal{L}_{c} \approx 1.5 R$, implying that only the lowest harmonics contribute significantly to $\chi_{\mathrm{sc}}^{\text {osc }}$. This may explain why the peak from the shortest orbits, the bouncing-ball orbits, is comparable to the other peaks. The influence of this first peak causes small deviations between the semiclassical and the quantum-mechanical result, as visible in Fig. 13(b). There, $\chi^{\text {osc }}$ is normalized by $\sqrt{k_{F} R}$ and

$$
X=\left(0.5 \phi_{0} / \mathcal{A}\right) \chi_{0} \approx-7.8 R \times 10^{-5} \text {. }
$$

Due to the divergent character of $\chi_{0}$ for small values of $\phi$ [see Eq. (25)], the amplitude of the oscillations in $\chi^{\text {osc }}$ 

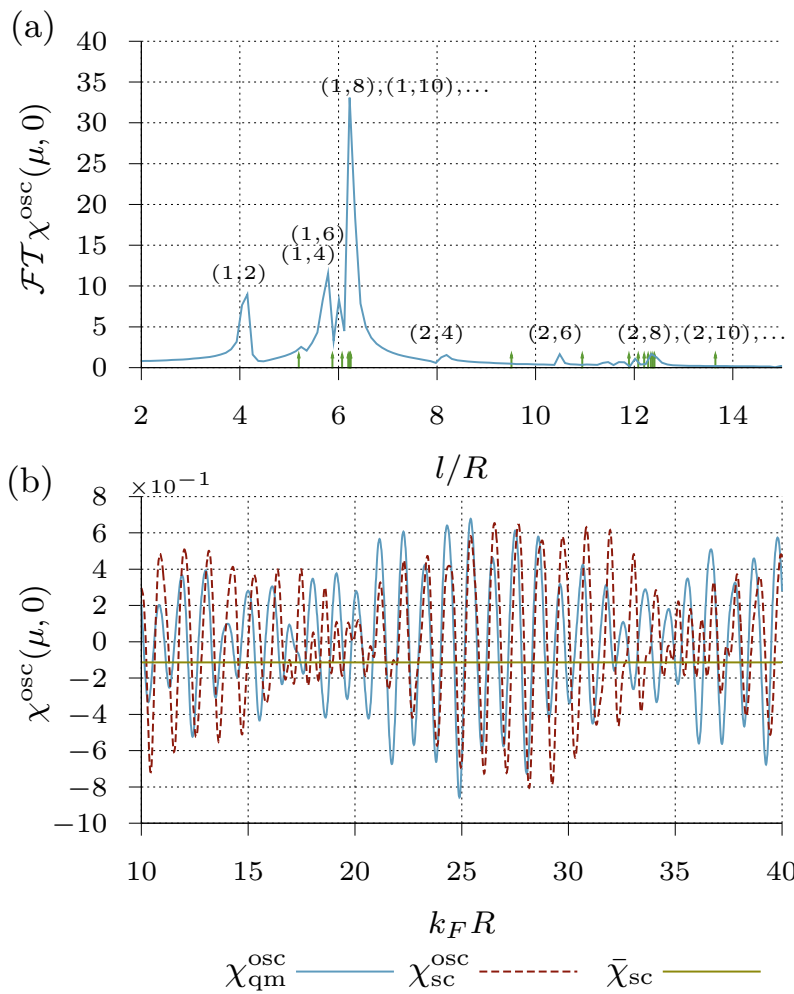

FIG. 13. (Color online) (a) Length spectrum of $\chi^{\text {osc }}$ calculated quantum mechanically from the eigenenergies of a graphene disk, Eq. (78), at $\phi=0,1 / \beta \approx 10^{-3} t$ and using $R \approx 200 a$. Peak positions correspond to orbit families $(w, v)$. Due to pseudospin interference, orbits with an odd number of reflections do not contribute. Their lengths are marked by green arrows. (b) Comparison of the semiclassical prediction (84) (red dashed line) for the orbital magnetic susceptibility with the quantum-mechanical result (solid blue) at $B=$ 0 and $1 / \beta \approx 10^{-3} t$. The (green) horizontal line represents the typical value, Eq. (88), of the magnetic susceptibility. All susceptibilities are normalized by $X=\chi_{0}\left[\phi_{0} /(2 \mathcal{A})\right]$ and $\sqrt{k_{F} R}$.

appears to be smaller than the contribution from the filled valence band. Anyhow, one would not expect the quantummechanical and the semiclassical result to lie in perfect agreement with each other since the susceptibility as a second derivative is very sensitive to small deviations already on the level of the DOS. The length spectrum Fig. 13(a) shows an accumulation of contributing orbits in the vicinity of $2 \pi w$. This clustering of orbit families can be identified with the so-called "whispering gallery" modes, which yield a coherent contribution to $\rho_{\mathrm{sc}}^{\mathrm{osc}}$. Since these orbit families enclose nearly the whole disk area, i.e., $\mathcal{A}_{w, v} \approx \mathcal{A} w$, their contribution to $\chi_{\mathrm{sc}}^{\mathrm{osc}}$ converges as $(-1)^{v / 2} / v^{2}$ for a fixed value of $w$ leading to an overall convergence of Eq. (83) at finite temperatures [16].

As has been done for corresponding systems of parabolic dispersion [16] one can calculate the typical value of the oscillatory susceptibility contribution defined by the root mean square of $\chi_{\mathrm{sc}}^{\text {osc }}$ with respect to energy [16]:

$$
\bar{\chi}_{\mathrm{sc}}(\mu, B)=\sqrt{\left\langle\left[\chi_{\mathrm{sc}}^{\mathrm{osc}}(\mu, B)\right]^{2}\right\rangle}
$$

with

$$
\left\langle\left[\chi_{\mathrm{sc}}^{\mathrm{osc}}(\mu, B)\right]^{2}\right\rangle=\frac{1}{\Delta k_{F} R} \int_{k_{F} R}^{k_{F} R+\Delta k_{F} R} d k_{F}^{\prime} R\left[\chi_{\mathrm{sc}}^{\mathrm{osc}}\left(E_{F}^{\prime}, B\right)\right]^{2} .
$$

The energy interval $\left[k_{F} R, k_{F} R+\Delta k_{F} R\right]$ is chosen classically negligible but quantum mechanically large, i.e., $k_{F} R \gg$ $\Delta k_{F} R \gg 2 \pi$. As a consequence, semiclassical off-diagonal terms $\propto \sin \left(k_{F} \mathcal{L}_{w, v}\right) \sin \left(k_{F} \mathcal{L}_{w^{\prime}, v^{\prime}}\right)$, where $(w, v) \neq\left(w^{\prime}, v^{\prime}\right)$, vanish under integration in Eq. (87), whereas the diagonal terms yield a contribution of $1 / 2$. For a detailed discussion see Ref. [16]. In the zero-field limit Eq. (86) simplifies to

$$
\begin{aligned}
\bar{\chi}_{\mathrm{sc}}(\mu, 0)= & -X \sqrt{k_{F} R} \times \frac{8 \sqrt{\pi}}{3 \zeta\left(\frac{3}{2}\right)} \\
& \times\left[\frac{1}{2} \sum_{\substack{w \\
v>2 w, \text { even }}} \frac{R_{T}^{2}\left(\frac{\mathcal{L}_{w, v}}{\hbar v_{F}}\right)}{v^{4}} \frac{\left(\mathcal{A}_{w, v} / R^{2}\right)^{4}}{\mathcal{L}_{w, v} / R}\right]^{1 / 2}
\end{aligned}
$$

in terms of $X$, Eq. (85). Choosing a similar cutoff length as in Fig. 13 , i.e., $\mathcal{L}_{c}=1.5 R$, yields $\bar{\chi}_{\mathrm{sc}}(\mu, 0) \approx-0.11 X \sqrt{k_{F} R}$, marked as a horizontal line in Fig. 13(b). In contrast to that, a calculation [16] yields for a circular quantum dot with parabolic dispersion $\bar{\chi}_{\mathrm{sc}, 2 \mathrm{DEG}} \approx 0.87 \chi_{L}\left(k_{F} R\right)^{3 / 2}$, where the Landau susceptibility $\chi_{L}$, Eq. (26), corresponds to $\chi_{0}$ in graphene.

We additionally considered ring-shaped graphene billiards of various thickness with infinte-mass-type edges. As shown in Ref. [60] this geometry can be quantized in Dirac approximation for arbitrary magnetic-field strength yielding a condition similar to Eq. (78). The comparison of $\chi_{\mathrm{qm}}^{\mathrm{osc}}$ with $\chi_{\mathrm{sc}}^{\mathrm{osc}}$ does not yield convincing coincidence in that case due to additional diffraction effects at the inner disk. These effects are beyond the leading-order semiclassical expansion considered in this work.

\section{Rectangular billiard with zigzag and armchair edges}

The second fundamental system we consider is a rectangular graphene quantum dot with zigzag edges in the $x$ direction and armchair edges in the $y$ direction similar to that in Fig. 14. The side lengths are labeled as $\mathcal{L}_{z z}$ and $\mathcal{L}_{a c}$, respectively, such that $\mathcal{A}=\mathcal{L}_{a c} \mathcal{L}_{z z}$. Similar to the comparable Schrödinger system, the Dirac equation for a rectangular graphene quantum dot cannot be solved analytically in the presence of a magnetic field. For this reason, we will calculate the eigenenergies numerically within tight-binding approximation to check the quality of the semiclassical prediction.

From Fig. 14 it is clear that opposite zigzag edges are built from different sublattices and lead to an additional sign of the reflection angle at one of the zigzag edges, as mentioned in Sec. IV. The classical periodic paths in this system can be classified by the tuple $(M, N)=(r m, r n)$ of their primitive reflection numbers $m$ and $n$ on the edges and their number of repetitions $r$. The corresponding orbit has $M$ bounces at the armchair and $N$ bounces at the zigzag edges in total and closes after $r$ repetitions. Figure 15 shows as examples members of the family $(1,1)$ and $(1,2)$, respectively. The members of one 


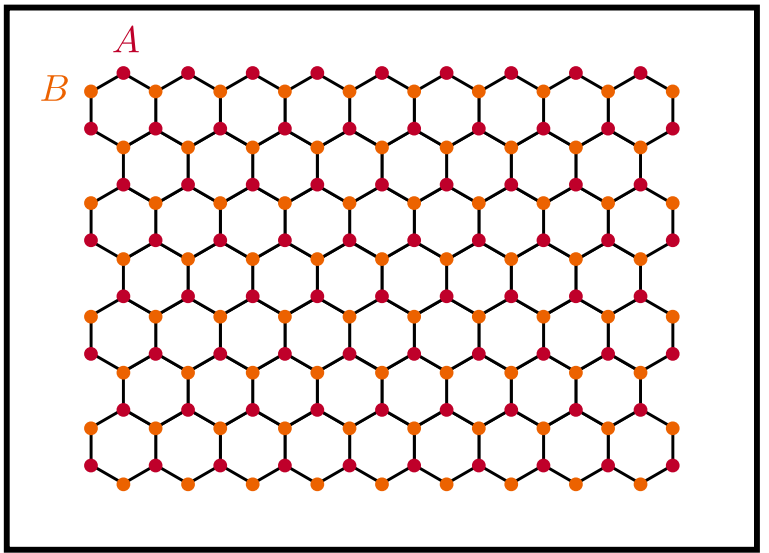

FIG. 14. (Color online) Example of a typical rectangular graphene quantum dot with $\mathcal{L}_{a c}=11 / \sqrt{3} a$ in $x$ and $\mathcal{L}_{z z}=9 a$ in $y$ direction.

orbit family can be transformed into each other via translation of the reflection point $x_{0} \in\left[0, \mathcal{L}_{\mathrm{zz}} / n\right]$ at the $x$ axis. Thus, all members of one family have the same path length $[16,36]$,

$$
\mathcal{L}_{M, N}=2 r \sqrt{\left(m \mathcal{L}_{z z}\right)^{2}+\left(n \mathcal{L}_{a c}\right)^{2}},
$$

and only the enclosed area depends on the translational group element $x_{0}$. From Refs. [16,36] follows

$$
\mathcal{A}_{M, N}\left(x_{0}\right)= \begin{cases}\frac{2 r}{m} \mathcal{L}_{a c} x_{0}\left(1-\frac{x_{0}}{\mathcal{L}_{z z}} n\right) & \text { if } m \cdot n \text { odd, } \\ 0 & \text { if } m \cdot n \text { even. }\end{cases}
$$

As visible in Fig. 15, the directed area does not vanish if $m \cdot n$ is odd. The flux-dependent dephasing factor reads

$$
\begin{aligned}
\mathcal{C}_{M, N}(B)= & \frac{n}{\mathcal{L}_{z z}} \int_{0}^{\mathcal{L}_{z z} / n} d x_{0} \cos \left(\frac{\mathcal{A}_{M, N}\left(x_{0}\right)}{l_{B}^{2}}\right) \\
= & \frac{\sqrt{\pi / 2}}{\sqrt{\phi_{M, N}}}\left[\cos \left(\phi_{M, N}\right) \mathrm{C}\left(\sqrt{\phi_{M, N}}\right)\right. \\
& \left.+\sin \left(\phi_{M, N}\right) \mathrm{S}\left(\sqrt{\phi_{M, N}}\right)\right] .
\end{aligned}
$$

The cosine Fresnel integral [48] $C(x)=\sqrt{2 / \pi} \int_{0}^{x} d t \cos \left(t^{2}\right)$ is defined analogous to $S(x)$ in Sec. IIIC. The phase

$$
\phi_{M, N}=\frac{\mathcal{A}_{M, N}\left[\mathcal{L}_{z z} /(2 n)\right]}{l_{B}^{2}}=\pi \frac{r}{m n} \varphi
$$
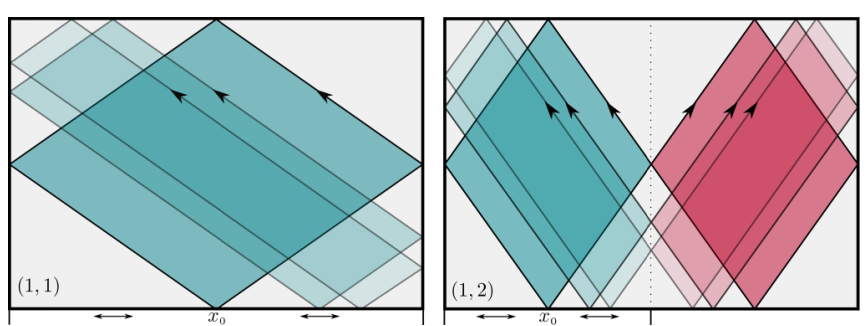

FIG. 15. (Color online) Three representative members of the orbit families $(1,1)$ and $(1,2)$. The enclosed area varies depending on the position of the reflection point $x_{0} \in\left[0, \mathcal{L}_{z z} / n\right]$ at the lower boundary. In the case of the $(1,2)$ family the net directed enclosed area is zero due to opposite propagation direction along both trajectory parts. corresponds to $2 \pi$ times the flux through the area $\mathcal{A}_{M, N}\left[\mathcal{L}_{z z} /(2 n)\right]=\mathcal{A} r /(2 m n)$, which is enclosed by the timereversed orbit partner with bounces at $x_{0}=\mathcal{L}_{z z} /(2 n)$. It can be directly proven that the enclosed area of these two orbits of the $(M, N)$ orbit family is maximum and therefore the action [Eq. (68)] stays extremal only for these two paths. Corresponding to the Poincaré-Birkhoff theorem, these are the only members of the orbit family which remain periodic in the presence of the perpendicular magnetic field [16]. In contrast to rotational symmetric systems, the magnetic field factor is not only governed by the dephasing between time-reversed orbit twins but also due to dephasing of family members propagating in the same direction.

The trace over the pseudospin-propagator is $[21,57]$

$$
\operatorname{Tr} K_{M, N}=g(-1)^{r n} \cos \left(2 K \mathcal{L}_{z z} r m-2 r n\left|\theta_{z z}\right|\right),
$$

where $K=4 \pi /(3 a)$ is the distance between the $\Gamma$ point and one of the $K$ points in the first Brillouin zone. The reflection angle $\left|\theta_{z z}\right|=\arctan \left[M \mathcal{L}_{z z} /\left(N \mathcal{L}_{a c}\right)\right]$ appears in Eq. (94) because the opposing zigzag edges are built from different sublattices (Fig. 14). On a microscopic scale the distance between both armchair edges can only take values $\mathcal{L}_{z z}=q \cdot a / 2, q \in \mathbb{N}$, yielding [21,57]

$$
K \mathcal{L}_{z z}= \begin{cases}0 \bmod 2 \pi, & \text { if } q \bmod 3=0, \\ \pi / 3 \bmod 2 \pi, & \text { otherwise }\end{cases}
$$

Whenever the length of the zigzag edge is such that $q$ is a multiple of 3 , orbit families with $\left(N \mathcal{L}_{a c} / \mathcal{L}_{z z}, N\right)$, where $N$ is odd, are suppressed by the trace of the pseudospin-propagator and hence do not contribute to the DOS [21,57]. Furthermore, when $\mathcal{L}_{a c}=\mathcal{L}_{z z}$ on a macroscopic scale, bouncing-ball orbits with $(0, M)$ and $(N, 0)$ cancel each other exactly if $M$ and $N$ are odd [21,57], respectively. Combining these considerations with the results for a rectangular Schrödinger system $[16,36]$ we find the field-dependent expression

$$
\begin{aligned}
\rho_{\mathrm{sc}}^{\text {osc }}(E, B)= & \frac{\mathcal{A}}{\hbar v_{F}} \sqrt{\frac{k}{2 \pi^{3}}} \sum_{r=1}^{\infty} \sum_{\substack{m, n=0 \\
m=0 \vee n=0}}^{\infty} \frac{f_{n, m}}{\sqrt{\mathcal{L}_{M, N}}} \\
& \times \cos \left(k \mathcal{L}_{M, N}-\frac{\pi}{4}\right) \operatorname{Tr} K_{M, N} \mathcal{C}_{M, N}(B) .
\end{aligned}
$$

The factor $f_{M, N}=2$ whenever there exists a time-reversed version of the orbit family $(M, N)$ and $f_{M, N}=1$ for bouncingball orbits. In order to calculate $\chi_{\mathrm{sc}}^{\mathrm{osc}}$ we take the second derivative of the field factor,

$$
\mathcal{C}_{M, N}^{\prime \prime}(B)=-\left(\frac{2 \pi \mathcal{A}_{M, N}}{\phi_{0}}\right)^{2} \frac{\sqrt{\pi / 2}}{4} \times \tilde{\mathcal{C}}_{M, N}\left(\phi_{M, N}\right),
$$

with $\phi_{M, N}$ in Eq. (93) and

$$
\begin{aligned}
\tilde{\mathcal{C}}_{M, N}(x)= & \sqrt{\frac{2}{\pi}} \frac{3}{x^{2}}-\frac{C(\sqrt{x})}{x^{5 / 2}}\left[\left(3-4 x^{2}\right) \cos (x)+4 x \sin (x)\right] \\
& -\frac{S(\sqrt{x})}{x^{5 / 2}}\left[\left(3-4 x^{2}\right) \sin (x)-4 x \cos (x)\right] .
\end{aligned}
$$


In the zero-field limit $\tilde{C}_{M, N}$ converges to the value $(32 / 15) \sqrt{2 / \pi}$. For $\chi_{\text {sc }}^{\text {osc }}$ we find, according to Eq. (77),

$$
\begin{aligned}
\chi_{\mathrm{sc}}^{\mathrm{osc}}(\mu, B)= & -\chi_{0}(B) \times \frac{\sqrt{2} 2 \pi}{3 \zeta(3 / 2)}\left(\frac{\mathcal{L}_{z z}}{\mathcal{L}_{a c}}\right)^{2} \frac{\mathcal{L}_{a c}}{l_{B}} \sqrt{k_{F} \mathcal{L}_{a c}} \\
& \times \sum_{\substack { r=1 \\
\begin{subarray}{c}{m, n=1 \\
m \cdot n \text { odd }{ r = 1 \\
\begin{subarray} { c } { m , n = 1 \\
m \cdot n \text { odd } } }\end{subarray}}^{\infty} \frac{\operatorname{Tr} K_{M, N}}{g}\left(\frac{\mathcal{A}_{M, N}}{\mathcal{A}}\right)^{2} \sqrt{\frac{\mathcal{L}_{a c}}{\mathcal{L}_{M, N}}} \\
& \times \cos \left(k_{F} \mathcal{L}_{M, N}-\frac{\pi}{4}\right) R_{T}\left(\frac{\mathcal{L}_{M, N}}{\hbar v_{F}}\right) \tilde{\mathcal{C}}_{M, N}\left(\phi_{M, N}\right) .
\end{aligned}
$$

The factor $f_{M, N}=2$ for all contributing orbit families is absorbed in the prefactor. The squared aspect ratio $\mathcal{L}_{z z} / \mathcal{L}_{a c}$ enters the prefactor yielding a strong dependence of the susceptibility on the geometry of the system.

Panels (a) and (c) of Fig. 16 show the length spectra obtained after Fourier transform from $\chi^{\text {osc }}$ calculated from the tight-binding eigenenergies of two rectangular graphene quantum dots with $\mathcal{L}_{a c}=201.207 a, \mathcal{L}_{z z}=201 a$ and $\mathcal{L}_{a c}=$ $202.073 a, \mathcal{L}_{z z}=202 a$, respectively. The green arrows mark the position of orbit families which are semiclassically predicted not to contribute to $\chi^{\text {osc }}$. These length spectra are not as smooth as the one obtained for the graphene disk with infinite mass boundaries, Fig. 13(a), since the region of linear dispersion cannot be extended arbitrarily in tight-binding approximation. Still, one can clearly identify the peaks in Figs. 16(a) and 16(c) with the lengths of contributing orbits, such that the comparison of $\chi_{\mathrm{TB}}^{\mathrm{osc}}$, with $\chi_{\mathrm{sc}}^{\text {osc }}$, Eq. (99), in Figs. 16(b) and 16(d) shows convincing agreement. In these cases the thermal energy is $1 / \beta=10^{-3} t$ corresponding to the cutoff length $\mathcal{L}_{c} \approx 1.5 \mathcal{L}_{a c}$. The normalization factor $X / \sqrt{k_{F} \mathcal{L}_{a c}}$ is the same as the one chosen in Sec. IV C, with $X$ defined by Eq. (85) and $\mathcal{L}_{\text {ac }} \approx R$, i.e., all parameters are similar to the disk. The amplitudes of the oscillation between para- and diamagnetic behavior of $\chi^{\text {osc }}$ in the case of the rectangular quantum dots [Figs. 16(b) and 16(d)] are similar to the amplitude of $\chi^{\text {osc }}$ for the circular quantum dot [Fig. 13(b)]. Though the agreement of the oscillation frequencies of $\chi_{\mathrm{TB}}^{\mathrm{osc}}$ and $\chi_{\mathrm{sc}}^{\mathrm{osc}}$ in Figs. 16(b) and 16(d) are convincing the tight-binding result in panel (d) exhibits an additional modulation of the oscillations for $k_{F} \mathcal{L}_{a c}>22$ which are not contained in the semiclassical approximation. In the corresponding energy range, the Dirac model and therefore the semiclassical approximation reaches the limit of validity [57] in describing the energy spectrum of graphene when $k a \lesssim 1$. Though the lengths $\mathcal{L}_{a c, z z}$ of the system considered in Figs. 16(a) and 16(b) $\left(K \mathcal{L}_{z z}=0 \bmod 2 \pi\right)$ are only one row of atoms shorter on each side than the system considered in Figs. $16(\mathrm{c})$ and $16(\mathrm{~d})\left(K \mathcal{L}_{z z}=\pi / 3 \bmod 2 \pi\right)$, the oscillation amplitude of $\chi^{\text {osc }}$ differs by one order of magnitude. This is due to the suppression of orbit families with $\left(N \mathcal{L}_{a c} / \mathcal{L}_{z z}, N\right)$, where $N$ is odd, as noted above. Since the aspect ratio is not perfectly integer, those orbit families still yield a small contribution to $\rho^{\text {osc }}$, and correspondingly to $\chi^{\text {osc }}$, and appear in the length spectrum, Fig. 16(b).
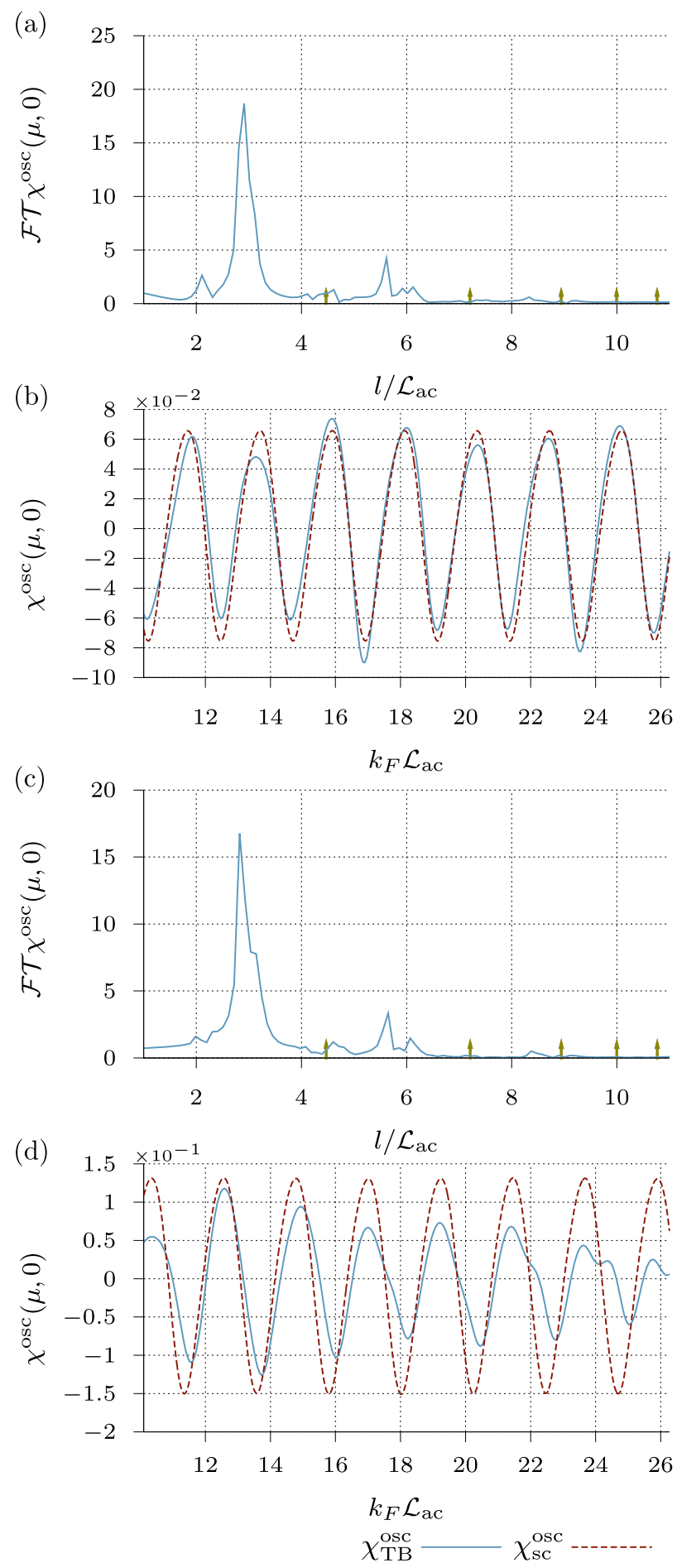

FIG. 16. (Color online) Panels (a) and (b): Length spectra calculated from the Fourier transform of the tight-binding results for $\chi^{\text {osc }}$; panels (b) and (d): Comparison of $\chi^{\text {osc }}$ in semiclassical approximation with corresponding numerical tight-binding results. Results shown in (a) and (b) are obtained for a rectangular graphene quantum dot with $K \mathcal{L}_{z z}=0 \bmod 2 \pi$ and side lengths of $\mathcal{L}_{z z}=201 a$ and $\mathcal{L}_{a c} \approx 201.207 a$, respectively. Panels (c) and (d) display results for a cavity with side lengths $\mathcal{L}_{z z}=202 a$ and $\mathcal{L}_{a c} \approx 202.073 a$ such that $K \mathcal{L}_{z z}=\pi / 3 \bmod 2 \pi$. Green arrows in panels (a) and (c) mark positions of orbits that do not contribute to the susceptibility due to their vanishing directed enclosed area $\mathcal{A}_{M, N} \cdot \chi^{\text {osc }}$ is normalized by $X / \sqrt{k_{E} \mathcal{L}_{a c}}$ in all cases, with $X$ as defined in Eq. (85). 


\section{SUMMARY AND OUTLOOK}

In this work we focused on the orbital magnetic properties of noninteracting ballistic bulk graphene and in particular on confined graphene-based systems with regular classical dynamics. To this end we considered the magnetic susceptibility $\chi$, calculated in the grand canonical ensemble, in the energy region of linear dispersion.

In the first part of this paper, we considered bulk graphene. There the orbital magnetic response distinctly depends on the particular energy scales involved, namely the different energy regimes associated with temperature, chemical potential, and magnetic field that we considered with a comparative look.

In a first step we derived the temperature-independent susceptibility contribution $\chi_{0}$ from the filled valence band, i.e., the graphene analog to the Landau susceptibility $\chi_{L}$ of an ordinary two-dimensional electron gas. We found for $\chi_{0}$ the well-known diamagnetic $-B^{-1 / 2}$ behavior, assuming, in accordance with literature, the valence band to be linear and extended, so $\chi_{0}$ cannot be directly compared to realistic tightbinding calculations even for very large systems. Still, for finite temperatures we found the total orbital magnetic susceptibility $\chi=\chi_{0}+\chi_{T}$ to be regular in the limit $B \rightarrow 0$ for $T \neq 0$. We compared our analytic results for the temperature-dependent part $\chi_{T}$ of the susceptibility with the results from literature and to numerical tight-binding calculations. The latter were performed for finite nanostructures of mesoscopic dimensions, also in view of the confinement effects later addressed. Still, the magnetic response of these graphene cavities also exhibits bulklike features, and we discussed initially the necessary conditions for comparison of our analytic bulk calculations with results for finite systems.

In the presence of a finite magnetic field the features of the susceptibility depend on the relative size of the associated Landau-level spacing $\Delta_{L L}$, the chemical potential $\mu$, and the thermal energy $k_{\mathrm{B}} T$. If the latter is the smallest scale, we distinguish two regimes (see Fig. 1):

For $\mu>\Delta_{L L}$ we obtained the typical $\mu^{2}$ - and $1 / B$ equidistant, oscillatory behavior of $\chi_{T}$, similar to de Haasvan Alphen oscillations in two-dimensional electron gases. Though the corresponding numerically calculated magnetic response for the finite systems exhibits, as a signature of the confinement, a richer oscillatory structure in this regime, there is a clear coincidence of clustered peaks with the pattern of $\chi_{T}$ in the bulk system. This becomes even more obvious by raising the temperature in the numerics, since then the finite-size contributions are damped out and only Landau level signatures remain. The amplitudes of these de Haas-van Alphen-type oscillations in graphene are one order of magnitude larger than the diamagnetic $\chi_{0}$ for the considered parameters, implying that the total orbital magnetic susceptibility oscillates between para- and diamagnetic behavior as a function of $\mu$ and $B$, respectively.

For $\mu<\Delta_{L L}$ the term $\chi_{T}$, and therefore $\chi$, is an exponentially decaying function of the magnetic field and diamagnetic. For field values high enough such that bulk effects dominate over finite-size signatures, the numerically calculated susceptibility of the quantum dots coincides very well with the analytic results.
If $k_{\mathrm{B}} T$ is larger than $\Delta_{L L}, \chi_{T}$ is a smooth function of temperature, chemical potential, and magnetic field and shows paramagnetic behavior with values $\lesssim 0.4\left|\chi_{0}\right|$. Therefore, $\chi=$ $\chi_{0}+\chi_{T}$ is diamagnetic and appears even to be independent of the magnetic field for arbitrary $\mu$. At the Dirac point $(\mu=0)$, $\chi_{T}$, and correspondingly $\chi$, follow a Curie-type $T^{-1}$ power law which is confirmed by our numerical data for the (triangular) quantum dots at finite temperature. For $1 / \beta \lesssim t$, deviations between $\chi_{T}$ for the bulk and the finite systems appear due to the increasing relevance of finite-size signatures in this limit. We also analytically confirmed the well-known $\delta(\mu)$ singularity $[2,4-11]$ of $\chi$ at zero temperature.

Through the confirmation of the analytic results for extended graphene with numerical data of finite quantum dots, we could analyze the importance of bulk effects in a finite system on the one hand and distinguish them from true confinement effects on the other hand. As one interesting aspect we found $\chi_{T} / \chi_{0}$ of the triangular quantum dot with zigzag edges to be smaller than $\chi_{T} / \chi_{0}$ for the armchair quantum dot with same parameters. This is due to the zigzag edge state and the lower average energy in that case. Moreover, especially in the energy range, where oscillations occur in $\chi_{T}$, the influence of the boundary is clearly observable.

In the second, major part of this work we then analyzed in detail such confinement effects. To this end we considered two representative geometries, a disk-shaped and a rectangular graphene cavity. We derived a generic analytic expression for the oscillatory part $\chi^{\text {osc }}$ of the orbital magnetic susceptibility based on results [16] for the susceptibility of confined electron gases and working out generalizations to finite $B$ fields of semiclassical expressions for the field-free density of states for graphene cavities, Refs. [20,21,57]. We demonstrated that graphene specific edge effects depending on the type of the boundaries enter the semiclassical expressions, and thereby orbital magnetism, through phases associated with the pseudospin propagator. This semiclassical approximation applies in particular to the low-field regime, where bulk contributions are suppressed and the energy spectrum (and correspondingly the orbital susceptibility) is governed by finite-size effects.

We found good agreement of our semiclassical approach with the quantum-mechanical results for $\chi^{\text {osc }}$ based on the calculation of the eigenenergies for circular graphene quantum dots with infinite-mass type edges. The Fourier transform of $\chi_{\mathrm{qm}}^{\mathrm{osc}}$ with respect to the energy yielded a length spectrum with relatively sharp peaks reflecting the underlying classical orbit dynamics of this system. We showed that orbits with odd number of reflections are suppressed as it is predicted in our semiclassical approach due to destructive pseudospin interferences. This is distinctly different from the corresponding case of the electron-gas system [16], where all non-self-retracing orbits yield a contribution to $\chi_{\mathrm{sc}}^{\text {osc }}$. We found the typical value for $\left|\chi^{\text {osc }}\right|$ to scale like $\sqrt{k_{F} R}$. Hence, similar as in Ref. [16] $\left|\chi^{\text {osc }}\right|$ can be larger than $X=\chi_{0}\left(0.5 \phi_{0} / \mathcal{A}\right)$. In contrast, the amplitudes of the $\chi^{\text {osc }}$ oscillations show the same scaling behavior, but appear to be of the same order of magnitude as $X$. Similar agreement was found for rectangular-shaped graphene quantum dots, where we compared the semiclassical predictions with numerical tight-binding calculations. Depending on the length of the 
zigzag edges, the strength of the oscillatory modulations in $\chi$ were found to differ by one order of magnitude due to destructive pseudospin interferences.

We studied the magnetic response for individual systems, including the typical susceptibility, within the grand canonical formalism. To compute the average response of an ensemble of nanostructures, a canonical treatment starting from the free energy instead of the grand potential is required [61]. Along the lines of $[16,22]$, and with the semiclassical expressions for graphene derived here, it appears straightforward to compute the ensemble-averaged susceptibility.

A further interesting aspect concerns the role of disorder for orbital magnetism in graphene, both for the bulk and confined case. Again, previous work [62,63] for the 2D Schrödinger case, covering the entire disorder range from clean to diffusive, could act as a guideline.

Our overall analysis demonstrates pronounced confinement effects on orbital magnetism in graphene-based nanosystems that dominate the bulk response in wide parameter regimes. However, our approach is based on noninteracting models for graphene, as most of the works on orbital magnetism in graphene. An exception is Ref. [64] where interaction effects are considered at $T=0$, however only to first order in the Coulomb repulsion. The physics of conventional two-dimensional electron systems shows that, while noninteracting terms are also crucial there, contributions from electron-electron interactions can usually not be disregarded, for instance, for the two-dimensional bulk Aslamazov and Larkin computed interaction corrections to the Landau susceptibility [65] (see also Ref. [66] for a semiclassical treatment). Moreover, this work demonstrated that higher-order diagrams are essential for an appropriate perturbative treatment of interaction effects, a treatment that is missing for graphene. In Ref. [67] it was furthermore shown that additional confinement-mediated interaction contributions to the susceptibility of $2 \mathrm{D}$ electron systems can be of the same order as those from the noninteracting model. To generalize such an analysis in terms of interaction effects for graphene is beyond the scope of the present work. Hence this interesting and challenging question is left for future research.

\section{ACKNOWLEDGMENTS}

We thank I. Adagideli and J. Wurm for useful discussions and V. Krückl for help in numerical implementations. This work was funded by the Deutsche Forschungsgemeinschaft through GRK 1570: Electronic Properties of Carbon Based Nanostructures.

\section{APPENDIX A: TRANSFORMATION OF THE FRESNEL INTEGRAL}

Starting with the definition of the Fresnel integral [48], $S(x)=\sqrt{2 / \pi} \int_{0}^{x} d t \sin \left(t^{2}\right)$, one finds after substituting $t^{2}=\tau$ and using the relation [48]

$$
\frac{1}{z^{\alpha}}=\frac{1}{\Gamma(\alpha)} \int_{0}^{\infty} d u \frac{e^{-z u}}{u^{1-\alpha}}, \quad \operatorname{Re} z>0, \operatorname{Re} \alpha>0,
$$

where $z=\tau$ and $\alpha=1 / 2$,

$$
\begin{aligned}
S(|x|) & =\frac{1}{\sqrt{2 \pi} \Gamma\left(\frac{1}{2}\right)} \operatorname{Im}\left[\int_{0}^{\infty} d u \frac{1}{\sqrt{u}} \int_{0}^{x^{2}} d \tau e^{-(u-i) \tau}\right] \text { (A2) } \\
& =\frac{1}{\sqrt{2} \pi} \operatorname{Im}\left[\int_{0}^{\infty} d u \frac{1}{\sqrt{u}(u-i)}-\int_{0}^{\infty} d u \frac{e^{-(u-i) x^{2}}}{\sqrt{u}(u-i)}\right] .
\end{aligned}
$$

The first term in Eq. (A3) yields $1 / 2$ and represents the smooth part of the Fresnel integral. Using [48]

$$
U(1-\alpha ; 1-\alpha ; x)=\frac{x^{\alpha}}{\Gamma(1-\alpha)} \int_{0}^{\infty} d t \frac{e^{-t} t^{-\alpha}}{t+x}
$$

one finds $\left(\alpha=1 / 2\right.$ and $\left.t=u x^{2}\right) S(|x|)=1 / 2+\tilde{\mathrm{S}}(x)$ with

$$
\tilde{\mathrm{S}}(x)=-\frac{1}{\sqrt{2 \pi}} \operatorname{Im}\left[e^{i(\pi / 4)} e^{i x^{2}} U\left(\frac{1}{2} ; \frac{1}{2} ;-i x^{2}\right)\right] .
$$

\section{APPENDIX B: TRANSFORMATION OF $\tilde{\Omega}_{T}$ FOR $\alpha, \gamma>1$}

In order to calculate $\tilde{\Omega}_{T}$ as given in Eq. (48) for $\alpha, \gamma>1$ it is useful to apply the Taylor series representations of the logarithmic and exponential function yielding

$$
\begin{aligned}
\tilde{\Omega}_{T}-\hat{\Omega}_{T}= & g \frac{\varphi}{\beta} \sum_{s \pm 1} \sum_{\substack{n=1 \\
m=1}}^{\infty} \frac{(-1)^{m}}{m} e^{s(\gamma / \alpha) m} \\
& \times \sum_{k=0}^{\infty} \frac{(-1)^{k}}{\Gamma(k+1)}(\sqrt{2} \gamma m)^{k} n^{k / 2} .
\end{aligned}
$$

In the next step we interchange the order of summation [3], which can be done without causing correction terms in this particular situation [68]. Computing the sum over the Landau index $n$ first yields [48] $\sum_{n=1}^{\infty} n^{k / 2}=\zeta(-k / 2)$, where $\zeta(z)$ is the Riemann $\zeta$ function. With use of [48]

$$
\zeta(z)=\frac{1}{\Gamma(z)} \int_{0}^{\infty} d t \frac{t^{z-1}}{e^{t}-1}
$$

Eq. (B1) transforms to

$$
\begin{aligned}
\tilde{\Omega}_{T}-\hat{\Omega}_{T}= & g \frac{\varphi}{\beta} \sum_{s \pm 1} \sum_{m=1}^{\infty} \frac{(-1)^{m}}{m} e^{s(\gamma / \alpha) m} \\
& \times \sum_{k=0}^{\infty} \frac{(-1)^{k}}{\Gamma(k+1) \Gamma\left(-\frac{k}{2}\right)} \int_{0}^{\infty} d t \frac{\left(\frac{\sqrt{2} \gamma m}{\sqrt{t}}\right)^{k}}{t\left(e^{t}-1\right)} .
\end{aligned}
$$

We substitute $t=2 \gamma^{2} m^{2} \cdot y=u \cdot y$ such that the integral in Eq. (B3) can be approximated by

$$
\int_{0}^{\infty} d y \frac{y^{-k / 2-1}}{\exp (u y)-1} \stackrel{\gamma>1}{\approx} \int_{0}^{\infty} d y y^{-k / 2-1} e^{-u y}=\Gamma\left(-\frac{k}{2}\right) u^{k / 2} .
$$

Calculating subsequently the sums over $k$ and $m$ in Eq. (B3) finally yields

$$
\tilde{\Omega}_{T}-\hat{\Omega}_{T} \approx-g \frac{\varphi}{\beta} \sum_{s \pm 1} \ln \left[1+e^{-\sqrt{2} \gamma+s(\gamma / \alpha)}\right] .
$$


[1] L. Landau, Z. Phys. A 64, 629 (1930).

[2] J. W. McClure, Phys. Rev. 104, 666 (1956).

[3] A. Ghosal, P. Goswami, and S. Chakravarty, Phys. Rev. B 75, 115123 (2007).

[4] S. A. Safran and F. J. DiSalvo, Phys. Rev. B 20, 4889 (1979).

[5] M. Koshino and T. Ando, Phys. Rev. B 75, 235333 (2007).

[6] M. Nakamura, Phys. Rev. B 76, 113301 (2007).

[7] M. Koshino, Y. Arimura, and T. Ando, Phys. Rev. Lett. 102, 177203 (2009).

[8] Y. Arimura, M. Koshino, and T. Ando, J. Phys. Soc. Jpn. 80, 114705 (2011).

[9] A. Jellal, M. Bellati, and M. Schreiber, J. Phys. A 44, 275001 (2011).

[10] A. Scholz and J. Schliemann, Phys. Rev. B 83, 235409 (2011).

[11] A. Principi, M. Polini, and G. Vignale, Phys. Rev. B 80, 075418 (2009).

[12] V. Chandrasekhar, R. A. Webb, M. J. Brady, M. B. Ketchen, W. J. Gallagher, and A. Kleinsasser, Phys. Rev. Lett. 67, 3578 (1991).

[13] D. Mailly, C. Chapelier, and A. Benoit, Phys. Rev. Lett. 70, 2020 (1993).

[14] L. Levy, D. Reich, L. Pfeiffer, and K. West, Physica B 189, 204 (1993).

[15] U. Eckern and P. Schwab, Adv. Phys. 44, 387 (1995).

[16] K. Richter, D. Ullmo, and R. A. Jalabert, Phys. Rep. 276, 1 (1996).

[17] D. Ullmo, Rep. Prog. Phys. 71, 026001 (2008).

[18] A. C. Bleszynski-Jayich, W. E. Shanks, B. Peaudecerf, E. Ginossar, F. von Oppen, L. Glazman, and J. G. E. Harris, Science 326, 272 (2009).

[19] F. von Oppen and E. K. Riedel, Phys. Rev. B 48, 9170 (1993).

[20] J. Wurm, K. Richter, and I. Adagideli, Phys. Rev. B 84, 075468 (2011).

[21] J. Wurm, K. Richter, and I. Adagideli, Phys. Rev. B 84, 205421 (2011).

[22] D. Ullmo, K. Richter, and R. A. Jalabert, Phys. Rev. Lett. 74, 383 (1995)

[23] L. Onsager, Philos. Mag. 43, 1006 (1952).

[24] R. Peierls, Z. Phys. A 80, 763 (1933).

[25] R. A. Shepherd, M. Elliott, W. G. Herrenden-Harker, M. Zervos, P. R. Morris, M. Beck, and M. Ilegems, Phys. Rev. B 60, R11277 (1999).

[26] S. G. Sharapov, V. P. Gusynin, and H. Beck, Phys. Rev. B 69, 075104 (2004).

[27] S. Zhang, N. Ma, and E. Zhang, J. Phys.: Condens. Matter 22, 115302 (2010).

[28] R. Enderlein and N. Horing, Fundamentals Of Semiconductor Physics And Devices (World Scientific, Singapore, 1997).

[29] S. Slizovskiy and J. J. Betouras, Phys. Rev. B 86, 125440 (2012).

[30] R. Peierls, Z. Phys. A 81, 186 (1933).

[31] T. Ando, J. Phys. Soc. Jpn. 74, 777 (2005).

[32] R. S. Deacon, K.-C. Chuang, R. J. Nicholas, K. S. Novoselov, and A. K. Geim, Phys. Rev. B 76, 081406 (2007).

[33] G. Li and E. Andrei, Nat. Phys. 3, 623 (2007).

[34] C. Kittel, Introduction to Solid State Physics (Addison-Wesley, Boca Raton, 1995).
[35] A. Messiah, Quantum Mechanics (North-Holland, Amsterdam, 1961).

[36] M. Brack and R. K. Bhaduri, Semiclassical Physics (Westview, Boulder, 2003).

[37] A. H. Castro Neto, F. Guinea, N. M. R. Peres, K. S. Novoselov, and A. K. Geim, Rev. Mod. Phys. 81, 109 (2009).

[38] M. Wimmer, Ph.D. thesis, University of Regensburg, 2009.

[39] M. Wimmer and K. Richter, J. Comput. Phys. 228, 8548 (2009).

[40] C. Lanczos, J. Res. Nat. Bur. Stand. 45, 255 (1950).

[41] R. Lehoucq, K. Maschhoff, D. Sorensen, and C. Yang, ARPACK.

[42] M. Zarenia, A. Chaves, G. A. Farias, and F. M. Peeters, Phys. Rev. B 84, 245403 (2011).

[43] F. Libisch, S. Rotter, J. Güttinger, C. Stampfer, and J. Burgdörfer, Phys. Rev. B 81, 245411 (2010).

[44] M. Robnik, J. Phys. A 19, 3619 (1986).

[45] P. John and L. G. Suttorp, J. Phys. A 28, 6087 (1995).

[46] J. Moré, Numerical Analysis, edited by G. Watson, Lecture Notes in Mathematics Vol. 630 (Springer, Berlin, 1978), pp. 105-116.

[47] Y. Ominato and M. Koshino, Phys. Rev. B 87, 115433 (2013).

[48] I. Gradshtein, I. Ryzhik, A. Jeffrey, and D. Zwillinger, Table of Integrals, Series and Products (Academic, New York, 2007).

[49] M. Gutzwiller, Chaos in Classical and Quantum Mechanics (Springer, New York, 1990).

[50] M. V. Berry and M. Tabor, J. Phys. A 10, 371 (1977).

[51] S. C. Creagh and R. G. Littlejohn, Phys. Rev. A 44, 836 (1991).

[52] S. C. Creagh, Ann. Phys. (N. Y.) 248, 60 (1996).

[53] O. Bohigas, M.-J. Giannoni, A. M. Ozorio de Almeida, and C. Schmit, Nonlinearity 8, 203 (1995).

[54] M. Brack, P. Meier, and K. Tanaka, J. Phys. A 32, 331 (1999).

[55] S. Tomsovic, M. Grinberg, and D. Ullmo, Phys. Rev. Lett. 75, 4346 (1995).

[56] D. Ullmo, M. Grinberg, and S. Tomsovic, Phys. Rev. E 54, 136 (1996).

[57] J. Wurm, Ph.D. thesis, University of Regensburg, 2011.

[58] A. Haar, Ann. Math.2nd Ser. 34, 147 (1933).

[59] M. V. Berry and R. J. Mondragon, Proc. R. Soc. London, Ser. A 412, 53 (1987).

[60] P. Recher, B. Trauzettel, A. Rycerz, Y. M. Blanter, C. W. J. Beenakker, and A. F. Morpurgo, Phys. Rev. B 76, 235404 (2007).

[61] Y. Imry, Introduction to Mesoscopic Physics, Mesoscopic Physics and Nanotechnology (Oxford University Press, New York, 1997).

[62] E. McCann and K. Richter, Europhys. Lett. 43, 241 (1998).

[63] E. McCann and K. Richter, Phys. Rev. B 59, 13026 (1999).

[64] A. Principi, M. Polini, G. Vignale, and M. I. Katsnelson, Phys. Rev. Lett. 104, 225503 (2010).

[65] L. G. Aslamazov and A. I. Larkin, Sov. Phys. JETP 40, 321 (1975).

[66] D. Ullmo, H. U. Baranger, K. Richter, F. von Oppen, and R. A. Jalabert, Phys. Rev. Lett. 80, 895 (1998).

[67] D. Ullmo, K. Richter, H. U. Baranger, F. von Oppen, and R. A. Jalabert, Physica E 1, 268 (1997).

[68] E. Elizalde, Ten Physical Applications of Spectral Zeta Functions, Lecture Notes in Physics, Vol. 855 (Springer, Berlin, 2012). 